Article

\title{
On Two Classes of Soft Sets in Soft Topological Spaces
}

\author{
Samer Al Ghour * and Worood Hamed \\ Department of Mathematics and Statistics, Jordan University of Science and Technology, Irbid 22110, Jordan; \\ whamed1971@gmail.com \\ * Correspondence: algore@just.edu.jo
}

Received: 16 January 2020; Accepted: 28 January 2020 ; Published: 9 February 2020

\begin{abstract}
In this paper, we define soft $\omega$-open sets and strongly soft $\omega$-open sets as two new classes of soft sets. We study the natural properties of these types of soft sets and we study the validity of the exact versions of some known results in ordinary topological spaces regarding $\omega$-open sets in soft topological spaces. Also, we study the relationships between the $\omega$-open sets of a given indexed family of topological spaces and the soft $\omega$-open sets (resp. strongly soft $\omega$-open sets) of their generated soft topological space. These relationships form a biconditional logical connective which is a symmetry. As an application of strongly soft $\omega$-open sets, we characterize soft Lindelof (resp. soft weakly Lindelof) soft topological spaces.
\end{abstract}

Keywords: $\omega$-open sets; soft Lindelof; soft weakly Lindelof; generated soft topology

\section{Introduction}

The soft set introduced by Molodtsov [1] is applied in many fields such as economics, engineering, social science, medical science, etc. It is used as a tool for dealing with uncertain objects. The theory of soft sets has been introduced and studied by several researchers (see [2,3]). Authors [1,4] applied soft sets in many areas such as Riemann integration, Perron integration, smoothness of function, operation research, game theory, probability and theory of measurements. Authors [5] applied soft sets in decision-making problems. The notion of soft topological spaces is introduced in [6]. Then researchers modified several concepts of classical topological spaces to include soft topological spaces, some recently published soft topological papers are appeared in [7-26]. For the purpose of improving some known topological theorems, Hdeib [27] introduced the notion of $\omega$-closed sets as a weaker notion of closed sets as follows: Let $(X, \Im)$ be a topological space and $A$ a subset of $X$. A point $x \in X$ is called a condensation point of $A$ if for each open set $U$ with $x \in U$, the set $U \cap A$ is uncountable. $A$ is called an $\omega$-closed subset of $X$ if it contains all its condensation points, $A$ is called an $\omega$-open subset of $X$ if $X-A$ is $\omega$-closed. As a known characterization of $\omega$-openness, $A$ is $\omega$-open if and only if for each $x \in A$ there exists $U \in \Im$ and a countable set $C \subseteq X$ such that $x \in U-C \subseteq A$. The family of all $\omega$-open subsets of $X$ is denoted by $\Im_{\omega}$. It is well known that $\Im_{\omega}$ forms a topology on $X$ finer than $\Im$. Also, it is known that the collection $\{U-C: U \in \Im$ and $C$ is a countable subset of $X\}$ is a base for $\Im$. Using $\omega$-open sets, Lindelöfness has been characterized in [27], several continuity concepts have been introduced and studied in [28-30], and several generalizations of paracompactness have been introduced and studied in [31]. Also, some modifications of both $\omega$-open and $\omega$-closed sets appear in [32-36]. The area of research related to $\omega$-open sets is still hot (see [37-43]), and the door is still open to use $\omega$-open for the purpose of generalizing some known topological concepts or improving some known topological results.

When we define a reasonable generalization of soft open sets in topological spaces, we hope that this will open the door for a number of future related research. For example, as a generalization of 
soft open sets in soft topological spaces, soft semiopen sets were defined in [44], then many related research articles have appeared, for instance, [45-50]. In this paper, we will modify the definition of $\omega$-open sets in classical topological spaces in order to define soft $\omega$-open sets and strongly soft $\omega$-open sets as two new classes of soft sets. We will study the natural properties of these types of soft sets, and we will study the validity of the exact versions of some known results in ordinary topological spaces regarding $\omega$-open sets in soft topological spaces. Also, we will study the relationships between the $\omega$-open sets of a given indexed family of topological spaces and the soft $\omega$-open sets of their generated soft topological space. These relationships form a biconditional logical connective which is a symmetry. As an application, we characterize soft Lindelof (resp. soft weakly Lindelof) soft topological spaces via strongly soft $\omega$-open sets.

This paper is organized as follows:

In Section 2, we introduce some basic definitions and results which we use them in our research.

In Section 3, we introduce and investigate soft $\omega$-open sets as a class of soft sets which contains the class of soft open sets.

In Section 4, we introduce and investigate strongly soft $\omega$-open sets as a class of soft sets which lies between the class of soft open sets and the class of soft $\omega$-open sets.

In Section 5, In this section, we use strongly soft omega open sets to characterize soft Lindelof soft topological spaces.

In Section 6, we define and investigate soft weakly Lindelof soft topological spaces. In particular, we characterize soft weakly Lindelof sets STS's which are strongly soft anti-locally countable via s $\omega$-open sets.

\section{Preliminaries}

In this section, we introduce some basic definitions and results which we use them in our paper.

Definition 1. [1] Let $X$ be an initial universe and $A$ be a set of parameters. $A$ soft set over $X$ relative to $A$ is a function $F: A \longrightarrow \mathcal{P}(X)$. The family of all soft sets over $X$ relative to $A$ will be denoted by $S S(X, A)$.

Definition 2. [2] Let $F, G \in S S(X, A)$. (1) $F$ is a soft subset of $G$, denoted by $F \widetilde{\subseteq} G$, if $F(a) \subseteq G(a)$ for each $a \in A$.

(2) $F$ and $G$ are said to be soft equal, denoted by $F=G$ if $F \widetilde{\subseteq} G$ and $F \widetilde{\subseteq} G$.

(3) Union of $F$ and $G$ is denoted by $F \widetilde{\cup} G$ and defined to be the soft set $F \widetilde{\cup} G \in S S(X, A)$ where $(F \widetilde{\cup} G)(a)=F(a) \cup G(a)$ for each $a \in A$.

(4) Intersection of $F$ and $G$ is denoted by $F \widetilde{\cap} G$ and defined to be the soft set $F \widetilde{\cap} G \in S S(X, A)$ where $(F \widetilde{\cap} G)(a)=F(a) \cap G(a)$ for each $a \in A$.

(5) The difference of $F$ and $G$ is denoted by $F-G$ and defined to be the soft set $F-G \in S S(X, A)$ where $(F-G)(a)=F(a)-G(a)$ for each $a \in A$.

Definition 3. [51] Let $\Delta$ be an arbitrary index set and $\left\{F_{\alpha}: \alpha \in \Delta\right\} \subseteq S S(X, A)$.

(a) The union of these soft sets is the soft set denoted by $\widetilde{\cup}_{\alpha \in \Delta} F_{\alpha}$ and defined by $\left(\underset{\alpha \in \Delta}{\widetilde{U}} F_{\alpha}\right)(a)=\bigcup_{\alpha \in \Delta} F_{\alpha}(a)$ for each $a \in A$.

(b) The intersection of these soft sets is the soft set denoted by $\widetilde{\bigcap_{\alpha \in \Delta}} F_{\alpha}$ and defined by $\left(\widetilde{\bigcap_{\alpha \in \Delta}} F_{\alpha}\right)(a)=$ $\bigcap_{\alpha \in \Delta} F_{\alpha}(a)$ for each $a \in A$.

Definition 4. [2] Let $F \in S S(X, A)$.

(a) $F$ is called a null soft set over $X$ relative to $A$, denoted by $0_{A}$, if $F(a)=\varnothing$ for each $a \in A$.

(b) $F$ is called an absolute soft set over $X$ relative to $A$, denoted by $1_{A}$, if $F(a)=X$ for each $a \in A$. 
Definition 5. [52] Let $F \in S S(X, A)$. F is called a soft point over $X$ relative to $A$ if there exist $e \in A$ and $x \in X$ such that

$$
F(a)=\left\{\begin{array}{cl}
\{x\} & \text { if } a=e \\
\varnothing & \text { if } a \neq e
\end{array} .\right.
$$

We denote $F$ by $e_{x}$. The family of all soft points over $X$ relative to $A$ is denoted by $S P(X, A)$.

Definition 6. [21] Let $X$ be an initial universe and let $A$ be a set of parameters. For any $a \in A$ and $Y \subseteq X$, the soft set $F \in S S(X, A)$ defined by

$$
F(b)= \begin{cases}Y & \text { if } b=a \\ \varnothing & \text { if } b \neq a\end{cases}
$$

will be denoted by $a_{Y}$.

Proposition 1. [52] Let $a_{x}, b_{y} \in S P(X, A)$. Then $a_{x}=b_{y}$ iff $x=y$ and $a=b$.

Definition 7. [52] Let $F \in S S(X, A)$ and $e_{x} \in S P(X, A)$. Then $e_{x}$ is said to belong to $F$ (notation: $e_{x} \widetilde{\in} F$ ) if $e_{x} \widetilde{\subseteq} F$ or equivalently: $e_{x} \widetilde{\in} F$ iff $x \in F(e)$.

Proposition 2. [21] $F \in S S(X, A)-\left\{0_{A}\right\}$ iff there is $a_{x} \in S P(X, A)$ such that $a_{x} \widetilde{\in} F$.

Proposition 3. [52] Let $F, G \in S S(X, A)$. Then the following are equivalent:

(a) $F \widetilde{\subseteq} G$.

(b) For all $a_{x} \in S P(X, A), a_{x} \tilde{\in} F$ implies $a_{x} \widetilde{\in} G$.

Definition 8. [52] Let $F \in S S(X, A)$. The set $\left\{a_{x}: a_{x} \widetilde{\in} F\right\}$ will be denoted by $P t(F)$.

It is clear that $S P(X, A)=P t\left(1_{A}\right)$.

Proposition 4. [52] Let $X$ be an initial universe and $A$ be a set of parameters. Then for any $F, G \in S S(X, A)$, $F \widetilde{\subseteq} G$ iff $P t(F) \subseteq P t(G)$.

Definition 9. [6] Let $\tau \subseteq S(X, A)$. Then $\tau$ is called a soft topology on $X$ relative to $A$ if

(1) $0_{A}, 1_{A} \in \tau$,

(2) the union of any number of soft sets in $\tau$ belongs to $\tau$,

(3) the intersection of any two soft sets in $\tau$ belongs to $\tau$.

The triplet $(X, \tau, A)$ is called a soft topological space (STS) over $X$ relative to $A$. The members of $\tau$ are called soft open sets in $(X, \tau, A)$ and their complements are called soft closed sets in $(X, \tau, A)$.

Proposition 5. [6] Let $(X, \tau, A)$ be a STS. Then the collection $\{F(a): F \in \tau\}$ defines a topology on $X$ for every $a \in A$. This topology will be denoted by $\tau_{a}$.

In this paper, the closure of a subset $Y \subseteq X$ in the topological space $\left(X, \tau_{a}\right)$ mentioned in Proposition 5 will be denoted by $\bar{Y}^{a}$.

Definition 10. [53] Let $(X, \tau, A)$ be a soft topological space. A subcollection $\mathcal{B}$ of $\tau$ is called a soft base of $\tau$ if every member of $\tau$ can be expressed as a union of members of $\mathcal{B}$.

Proposition 6. [54] Let $(X, \tau, A)$ be a STS and let $\mathcal{B} \subseteq \tau$. Then $\mathcal{B}$ is a soft base for $\tau$ if for every $F \in \tau-\left\{0_{A}\right\}$ and every $a_{x} \widetilde{\in} F$, there exists $G \in \mathcal{B}$ such that $a_{x} \widetilde{\in} G \widetilde{\subseteq} F$. 
Definition 11. [6] Let $(X, \tau, A)$ be a STS and let $G \in S S(X, A)$. The soft closure of $G$ in $(X, \tau, A)$ is denoted by $\mathrm{Cl}_{\tau}(G)$ and defined by

$$
C l_{\tau}(G)=\widetilde{\cap}\{F: F \text { is soft closed with } G \widetilde{\subseteq} F\} .
$$

Definition 12. [21] Let $X$ be an initial universe and let $A$ be a set of parameters. Let $\left\{\Im_{a}: a \in A\right\}$ be an indexed family of topologies on $X$. Then the soft topology $\left\{F \in S S(X, A): F(a) \in \Im_{a}\right.$ for all $\left.a \in A\right\}$ will be denoted by $\bigoplus_{a \in A} \Im_{a}$.

For any topological space $(X, \Im)$ and any set of parameters $A$, denote the family $\{F \in S S(X, A): F(a) \in \Im$ for all $a \in A\}$ by $\tau(\Im)$.

Proposition 7. [21] Let $X$ be an initial universe and let $A$ be a set of parameters. Let $\left\{\Im_{a}: a \in A\right\}$ be an indexed family of topologies on $X$. Then the family $\left\{a_{Y}: a \in A\right.$ and $\left.Y \in \Im_{a}\right\}$ is a soft base of $\bigoplus_{a \in A} \Im_{a}$.

Proposition 8. [21] Let $X$ be an initial universe and let $A$ be a set of parameters. Let $\left\{\Im_{a}: a \in A\right\}$ be an indexed family of topologies on $X$. Then $\left(\bigoplus_{a \in A} \Im_{a}\right)_{b}=\Im_{b}$ for all $b \in A$.

\section{Soft Omega Open Sets}

In this section, we introduce and investigate soft $\omega$-open sets as a class of soft sets which contains the class of soft open sets.

Definition 13. [52] Let $G \in S S(X, A)$. Then $G$ is called a countable soft set if for all $a \in A$, the set $G(a)$ is countable. The collection of all countable soft sets from SS $(X, A)$ will be denoted by $\operatorname{CSS}(X, A)$.

Definition 14. Let $(X, \tau, A)$ be a STS and let $G \in S S(X, A)$. Then $G$ is called a soft $\omega$-open set if for all $a_{x} \widetilde{\in}$ $G$, there exist $F \in \tau$ and $H \in \operatorname{CSS}(X, A)$ such that $a_{x} \widetilde{\in} F-H \widetilde{\subseteq} G$. The collection of all soft $\omega$-open sets in $(X, \tau, A)$ will be denoted by $\tau_{\omega}$.

Theorem 1. Let $(X, \tau, A)$ be a STS and let $G \in S S(X, A)$. Then $G$ is soft $\omega$-open if and only if for every $a_{x} \widetilde{\in}$ $G$ there exist $F \in \tau$ such that $a_{x} \tilde{\in} F$ and $F-G \in \operatorname{CSS}(X, A)$.

Proof. Necessity. Suppose that $G$ is soft $\omega$-open. Let $a_{x} \widetilde{\in} G$, then there exist $F \in \tau$ and $H \in \operatorname{CSS}(X, A)$ such that $a_{x} \widetilde{\in} F-H \widetilde{\subseteq} G$. Thus $a_{x} \widetilde{\in} F \in \tau$. Also, since $F-H \widetilde{\subseteq} G$, then $F-G \widetilde{\subseteq} H$ and so $F-G \in$ $\operatorname{CSS}(X, A)$.

Sufficiency. Suppose that for every $a_{x} \widetilde{\in} G$ there exist $F \in \tau$ such that $a_{x} \tilde{\in} F$ and $F-G \in$ $\operatorname{CSS}(X, A)$. Let $a_{x} \widetilde{\in} G$, then there exist $F \in \tau$ such that $a_{x} \widetilde{\in} F$ and $F-G \in \operatorname{CSS}(X, A)$. Put $H=$ $F-\left(G \widetilde{\cup} a_{x}\right)$. Then $H \in \operatorname{CSS}(X, A)$ and $a_{x} \widetilde{\in} F-H \widetilde{\subseteq} G$. It follows that $G$ is soft $\omega$-open.

Notation 1. For a STS $(X, \tau, A)$, denote the collection $\{F-H: F \in \tau$ and $H \in \operatorname{CSS}(X, A)\}$ by $\tau_{c}$.

Theorem 2. Let $(X, \tau, A)$ be a STS. Then
(a) $\tau \subseteq \tau_{c} \subseteq \tau_{\omega}$.
(b) $\left(X, \tau_{\omega}, A\right)$ is a STS.
(c) $\tau_{c}$ is a base for $\tau_{\omega}$.
(d) Countable soft sets are soft closed in $\left(X, \tau_{\omega}, A\right)$.

Proof. (a) Since $0_{A} \in \operatorname{CSS}(X, A)$, then $\tau=\left\{F-0_{A}: F \in \tau\right\} \subseteq \tau_{c}$. On the other hand, $\tau_{c} \subseteq \tau_{\omega}$ is obvious.

(b) (1) Since $0_{A}, 1_{A} \in \tau$, then by (a) $0_{A}, 1_{A} \in \tau_{\omega}$. 
(2) Let $F, G \in \tau_{\omega}$ and let $a_{x} \widetilde{\in} F \widetilde{\cap} G$. Then $a_{x} \widetilde{\in} F$ and $a_{x} \widetilde{\in} G$. Then by Theorem 1, there exist $H, W \in \tau$ such that $a_{x} \widetilde{\in} H \widetilde{\cap} W \in \tau$ and $H-F, W-G \in \operatorname{CSS}(X, A)$. It is not difficult to check that $(H \widetilde{\cap} W)-(F \widetilde{\cap} G)$. Thus by Theorem $1, F \widetilde{\cap} G \in \tau_{\omega}$.

(3) Let $\left\{G_{\alpha}: \alpha \in \Delta\right\} \subseteq \tau_{\omega}$ and let $a_{x} \widetilde{\in} \widetilde{\bigcup} G_{\alpha \in \Delta}$. Then there is $\beta \in \Delta$ such that $a_{x} \widetilde{\in} G_{\beta}$. So, there exist $F \in \tau$ and $H \in \operatorname{CSS}(X, A)$ such that $a_{x} \widetilde{\in} F-H \widetilde{\subseteq} G_{\beta} \widetilde{\subseteq} \widetilde{\bigcup} G_{\alpha \in \Delta}$. Therefore, $\widetilde{\bigcup}_{\alpha \in \Delta} G_{\alpha} \in \tau_{\omega}$.

(c) Obvious.

(d) Follows because by (a), $\tau_{c} \subseteq \tau_{\omega}$.

Theorem 3. Let $X$ be an initial universe, $A$ be a set of parameters and let $\tau=\left\{0_{A}\right\} \cup$ $\left\{1_{A}-H: H \in \operatorname{CSS}(X, A)\right\}$. Then $(X, \tau, A)$ is a STS.

Proof. (1) By definition of $\tau, 0_{A} \in \tau$. Since $0_{A} \in \operatorname{CSS}(X, A)$, then $1_{A}=\left(1_{A}-0_{A}\right) \in \tau$.

(2) Let $1_{A}-H, 1_{A}-K \in \tau-\left\{0_{A}\right\}$. Then $H \widetilde{\cup} K \in \operatorname{CSS}(X, A)$ and $\left(1_{A}-H\right) \widetilde{\cap}\left(1_{A}-K\right)=$ $\left(1_{A}-(H \widetilde{\cup} K)\right) \in \tau$.

(3) Let $\left\{1_{A}-H_{\alpha}: \alpha \in \Delta\right\} \subseteq \tau-\left\{0_{A}\right\}$. Then $\widetilde{\cap}\left\{H_{\alpha}: \alpha \in \Delta\right\} \in \operatorname{CSS}(X, A)$ and so $\widetilde{\cup}\left\{1_{A}-H_{\alpha}: \alpha \in \Delta\right\}=1_{A}-\widetilde{\cap}\left\{H_{\alpha}: \alpha \in \Delta\right\} \in \tau$.

Definition 15. Let $(X, \tau, A)$ be a STS. Then $\left\{0_{A}\right\} \cup\left\{1_{A}-H: H \in \operatorname{CSS}(X, A)\right\}$ will be called the cocountable soft topology and will be denoted by $\operatorname{coc}(X, A)$.

Proposition 9. For any STS $(X, \tau, A), \operatorname{coc}(X, A) \subseteq \tau_{c}$.

Proof. Obvious.

Theorem 4. For any STS $(X, \tau, A)$, the following are equivalent:
(a) $\operatorname{coc}(X, A) \subseteq \tau$.
(b) $\tau=\tau_{c}$.
(c) $\tau=\tau_{\omega}$.

Proof. (a) $\Longrightarrow$ (b): Suppose that $\operatorname{coc}(X, A) \subseteq \tau$. We need only to show that $\tau_{c} \subseteq \tau$. Let $G \in \tau$ and $H \in \operatorname{CSS}(X, A)$. Then $G-H=G \widetilde{\cap}\left(1_{A}-H\right)$. Since $\operatorname{coc}(X, A) \subseteq \tau$, then $1_{A}-H \in \tau$ and so $G-H \in \tau$. It follows that $\tau_{c} \subseteq \tau$.

(b) $\Longrightarrow$ (c): Suppose that $\tau=\tau_{c}$. Then $\tau_{c}$ is a soft topology. By Theorem 2 (c), it follows that $\tau_{c}=\tau_{\omega}$ and hence $\tau=\tau_{\omega}$.

(c) $\Longrightarrow$ (a): Suppose that $\tau=\tau_{\omega}$. Then by Proposition 9 and Theorem 2 (a), we have $\operatorname{coc}(X, A) \subseteq$ $\tau_{c} \subseteq \tau_{\omega}=\tau$.

Corollary 1. Let $X$ be an initial universe and $A$ be a set of parameters. Then $(\operatorname{coc}(X, A))_{\omega}=\operatorname{coc}(X, A)$.

Theorem 5. For any STS $(X, \tau, A)$ we have $\tau_{\omega}=\left(\tau_{\omega}\right)_{\omega}$.

Proof. By Proposition 9 and Theorem 2 (a), we have $\operatorname{coc}(X, A) \subseteq \tau_{c} \subseteq \tau_{\omega}$. Then by Theorem 4 , it follows that $\tau_{\omega}=\left(\tau_{\omega}\right)_{\omega}$.

Theorem 6. Let $(X, \tau, A)$ and $(X, \sigma, A)$ be two STS's. If $\tau \cup \operatorname{coc}(X, A) \subseteq \sigma$, then $\tau_{c} \subseteq \sigma$.

Proof. Let $F-H \in \tau_{c}$, where $F \in \tau$ and $H$ is a countable soft set. Since $F \in \tau, 1_{A}-H \in \operatorname{coc}(X, A)$ and $\tau \cup \operatorname{coc}(X, A) \subseteq \sigma$, then $F, 1_{A}-H \in \sigma$ and so $F \widetilde{\cap}\left(1_{A}-H\right)=F-H \in \sigma$.

Corollary 2. Let $(X, \tau, A)$ and $(X, \sigma, A)$ be two STS's. If $\tau \cup \operatorname{coc}(X, A) \subseteq \sigma$, then $\tau_{\omega} \subseteq \sigma$. 
Proof. Follows from Theorem 6 and Theorem 2 (c).

Lemma 1. [55] Let $(X, \tau, A)$ be a STS and let $\mathcal{B}$ be a soft base for $\tau$. Then for every a $\in$, the family $\{F(a): F \in \mathcal{B}\}$ forms a base for the topology $\tau_{a}$ on $X$.

Theorem 7. Let $(X, \tau, A)$ be a STS. Then for all $a \in A,\left(\tau_{a}\right)_{\omega}=\left(\tau_{\omega}\right)_{a}$.

Proof. Let $a \in A$. To show that $\left(\tau_{a}\right)_{\omega} \subseteq\left(\tau_{\omega}\right)_{a}$, it is sufficient to see that $U-C \in\left(\tau_{\omega}\right)_{a}$ for all $U \in \tau_{a}$ and a countable subset $C \subseteq X$. Let $U \in \tau_{a}$ and let $C$ be a countable subset of $X$. Since $U \in \tau_{a}$, then there is $F \in \tau$ such $F(a)=U$. Let $H=a_{C}$, then $H \in \operatorname{CSS}(X, A)$. Since we have $F-H \in \tau_{\omega}$, then $(F-H)(a)=F(a)-H(a)=U-C \in\left(\tau_{\omega}\right)_{a}$. To show that $\left(\tau_{\omega}\right)_{a} \subseteq\left(\tau_{a}\right)_{\omega}$, by Theorem 2 (c) and Lemma 1 it is sufficient to show that $\{(F-H)(a): F \in \tau$ and $H \in \operatorname{CSS}(X, A)\} \subseteq\left(\tau_{a}\right)_{\omega}$. Let $F \in \tau$ and $H \in \operatorname{CSS}(X, A)$, then $(F-H)(a)=F(a)-H(a)$ with $F(a) \in \tau_{a}$ and $H(a)$ is a countable subset of $X$ which implies that $(F-H)(a) \in\left(\tau_{a}\right)_{\omega}$.

Corollary 3. Let $(X, \tau, A)$ be a STS. If $G \in \tau_{\omega}$, then for all $a \in A$ we have $G(a) \in\left(\tau_{a}\right)_{\omega}$.

Proof. Let $G \in \tau_{\omega}$ and let $a \in A$. Then $G(a) \in\left(\tau_{\omega}\right)_{a}$ and by Theorem 7 we have $G(a) \in\left(\tau_{a}\right)_{\omega}$.

Lemma 2. [21] Let $X$ be an initial universe and let $A$ be a set of parameters. Let $\left\{\Im_{a}: a \in A\right\}$ be an indexed family of topologies on $X$. If $\mathcal{B}_{a}$ is a base for $\Im_{a}$ for all $a \in A$, then $\left\{a_{Y}: a \in A\right.$ and $\left.Y \in \mathcal{B}_{a}\right\}$ is a soft base of $\bigoplus_{a \in A} \Im_{a}$

Theorem 8. Let $X$ be an initial universe and let $A$ be a set of parameters. Let $\left\{\Im_{a}: a \in A\right\}$ be an indexed family of topologies on $X$. Then $\left(\bigoplus_{a \in A} \Im_{a}\right)_{\omega}=\bigoplus_{a \in A}\left(\Im_{a}\right)_{\omega}$.

Proof. To show that $\left(\bigoplus_{a \in A} \Im_{a}\right)_{\omega} \subseteq \bigoplus_{a \in A}\left(\Im_{a}\right)_{\omega}$, by Theorem 2 (c) it is sufficient to show that $\left(\bigoplus_{a \in A} \Im_{a}\right)_{c} \subseteq \bigoplus_{a \in A}\left(\Im_{a}\right)_{\omega}$. Let $F \in \bigoplus_{a \in A} \Im_{a}$ and $H$ be a countable soft set. Then for every $a \in A$, $F(a) \in \Im_{a}$ and $H(a)$ is a countable subset of $X$ and so $(F-H)(a)=F(a)-H(a) \in\left(\Im_{a}\right)_{\omega}$. Thus, $F-H \in \bigoplus_{a \in A}\left(\Im_{a}\right)_{\omega}$. For every $a \in A,\left\{U-C: U \in \Im_{a}\right.$ and $C$ is a countable subset of $\left.X\right\}$ is a base for $\left(\Im_{a}\right)_{\omega}$, so by Lemma $2\left\{a_{U-C}: a \in A, U \in \Im_{a}\right.$ and $C$ is a countable subset of $\left.X\right\}$ is a soft base for $\bigoplus_{a \in A}\left(\Im_{a}\right)_{\omega}$. Thus, to show that $\bigoplus_{a \in A}\left(\Im_{a}\right)_{\omega} \subseteq\left(\bigoplus_{a \in A} \Im_{a}\right)_{\omega}$ it is sufficient to show that $\left\{a_{U-C}: a \in A, U \in \Im_{a}\right.$ and $C$ is a countable subset of $\left.X\right\} \omega^{a} \subseteq$ $\left(\bigoplus_{a \in A} \Im_{a}\right)_{\omega}$. Note that $\left\{a_{U-C}: a \in A, U \in \Im_{a}\right.$ and $C$ is a countable subset of $\left.X\right\}=$ $\left\{a_{U}-a_{C}: a \in A, U \in \Im_{a}\right.$ and $C$ is a countable subset of $\left.X\right\}$, which ends the proof.

Lemma 3. [21] If $(X, \Im)$ is a topological space and $A$ is any set of parameters, then $(\tau(\Im))_{a}=\Im$ for all $a \in A$.

Corollary 4. If $(X, \Im)$ is a topological space and $A$ is any set of parameters, then $(\tau(\Im))_{\omega}=\tau\left(\Im_{\omega}\right)$ for all $a \in A$. 
Proof. For each $a \in A$, set $\Im_{a}=\Im$. Then $\tau(\Im)=\bigoplus_{a \in A} \Im_{a}$ and by Theorem 8

$$
\begin{aligned}
(\tau(\Im))_{\omega} & =\left(\bigoplus_{a \in A} \Im_{a}\right)_{\omega} \\
& =\bigoplus_{a \in A}\left(\Im_{a}\right)_{\omega} \\
& =\tau\left(\Im_{\omega}\right) .
\end{aligned}
$$

Definition 16. The STS $(X, \tau, A)$ is called a soft p-space if the countable intersection of soft open sets is soft open.

Definition 17. [56] A STS $(X, \tau, A)$ is called soft $T_{1}$ if for any two soft points $a_{x}, a_{y} \in S P(X, A)$ with $x \neq y$, there exist $G, F \in \tau$ such that $a_{x} \widetilde{\in} G-F$ and $a_{y} \widetilde{\in} F-G$.

Lemma 4. [56] A STS $(X, \tau, A)$ is soft $T_{1}$ if for every soft point $a_{x} \in \operatorname{SP}(X, A)$ is soft closed.

Theorem 9. If $(X, \tau, A)$ is soft $T_{1}$ and soft $p$-space, then $\tau=\tau_{\omega}$.

Proof. By Theorem 2 (a), $\tau \subseteq \tau_{\omega}$. To show that $\tau_{\omega} \subseteq \tau$, by Theorem 2 (c) it is sufficient to show that $\tau_{c} \subseteq \tau$. Let $F \in \tau$ and let $H \in \operatorname{CSS}(X, A)$. Since $(X, \tau, A)$ is soft $T_{1}$, then by Lemma $4 a_{x}$ soft closed for all $a_{x} \widetilde{\in} H$, and so $F-a_{x} \in \tau$ for all $a_{x} \widetilde{\in} H$. Since $(X, \tau, A)$ is soft $T_{1}$, then $\underset{a_{x} \widetilde{\in} H}{\widetilde{C}}\left(F-a_{x}\right) \in \tau$. Therefore,

$$
F-H=\underset{a_{x} \widetilde{\in} H}{\widetilde{C}}\left(F-a_{x}\right) \in \tau
$$

Definition 18. A STS $(X, \tau, A)$ is called soft locally countable if for $a_{x} \in \operatorname{SP}(X, A)$ there exists $G \in$ $\operatorname{CSS}(X, A) \cap \tau$ such that $a_{x} \widetilde{\in} G$.

Theorem 10. A STS $(X, \tau, A)$ is soft locally countable if and only if $\operatorname{SP}(X, A) \subseteq \tau_{c}$.

Proof. Necessity. Suppose that $(X, \tau, A)$ is soft locally countable. Let $a_{x} \in S P(X, A)$, then by soft local countability of $(X, \tau, A)$, there exists $G \in \operatorname{CSS}(X, A) \cap \tau$ such that $a_{x} \widetilde{\in} G$. Then $G-a_{x} \in \operatorname{CSS}(X, A)$ and so, $a_{x}=G-\left(G-a_{x}\right) \in \tau_{c}$.

Sufficiency. Suppose that $S P(X, A) \subseteq \tau_{c}$. Let $a_{x} \in S P(X, A)$. Then $a_{x} \in \tau_{c}$ and so there is $F \in \tau$ and $H \in \operatorname{CSS}(X, A)$ such that $a_{x}=F-H$. Then $F \in \operatorname{CSS}(X, A) \cap \tau$ with $a_{x} \widetilde{\in} F$. It follows that $(X, \tau, A)$ is soft locally countable.

Corollary 5. A STS $(X, \tau, A)$ is soft locally countable if and only if $\left(X, \tau_{\omega}, A\right)$ is a discrete STS.

Corollary 6. If $(X, \tau, A)$ is a STS with $X$ is countable, then $\left(X, \tau_{\omega}, A\right)$ is a discrete STS.

Theorem 11. For any STS $(X, \tau, A),\left(X, \tau_{\omega}, A\right)$ is soft $T_{1}$.

Proof. Follows from Theorem 2 (d) and Lemma 4.

Definition 19. [56] A STS $(X, \tau, A)$ is called soft $T_{2}$ if for any two soft points $a_{x}, a_{y} \in \operatorname{SP}(X, A)$ with $x \neq y$, there exist $G, F \in \tau$ such that $a_{x} \widetilde{\in} G, a_{y} \widetilde{\in} F$ and $G \widetilde{\cap} F=0_{A}$. 
Theorem 12. If $(X, \tau, A)$ is a soft $T_{2} S T S$, then $\left(X, \tau_{\omega}, A\right)$ is soft $T_{2}$.

Proof. Let $a_{x}, a_{y} \in S P(X, A)$ with $x \neq y$. Since $(X, \tau, A)$ is soft $T_{2}$, then there exist $G, F \in \tau$ such that $a_{x} \widetilde{\in} G, a_{y} \widetilde{\in} F$ and $G \widetilde{\cap} F=0_{A}$. By Theorem 2 (a), $\tau \subseteq \tau_{\omega}$ and so $G, F \in \tau_{\omega}$ which ends the proof that $\left(X, \tau_{\omega}, A\right)$ is soft $T_{2}$.

The following example shows that the converse of Theorem 2 need not to be true in general:

Example 1. Let $X=\mathbb{N}, A=\mathbb{R}$ and $\tau=\left\{1_{A}, 0_{A}\right\}$. By Corollary $5,\left(X, \tau_{\omega}, A\right)$ is a discrete STS. Thus, $\left(X, \tau_{\omega}, A\right)$ is soft $T_{2}$. On the other hand, it is clear that $(X, \tau, A)$ is not soft $T_{2}$.

Definition 20. A STS $(X, \tau, A)$ is called soft anti-locally countable if for every $F \in \tau-\left\{0_{A}\right\}, F \notin$ $\operatorname{CSS}(X, A)$.

Theorem 13. A STS $(X, \tau, A)$ is soft anti-locally countable if and only if $\left(X, \tau_{\omega}, A\right)$ is soft anti-locally countable.

Proof. Necessity. Suppose to the contrary that $(X, \tau, A)$ is soft anti-locally countable and there is $G \in \tau_{\omega}-\left\{0_{A}\right\}$ with $G \in \operatorname{CSS}(X, A)$. Choose $a_{x} \widetilde{\in} G$. There are $F \in \tau$ and $H \in \operatorname{CSS}(X, A)$ such that $a_{x} \widetilde{\in} F-H \widetilde{\subseteq} G$, and so $F \widetilde{\subseteq} H \widetilde{\cup} G$ which implies that $F \in \operatorname{CSS}(X, A)$. Since $F \in \tau-\left\{0_{A}\right\}$ and $(X, \tau, A)$ is soft anti-locally countable, then we have a contradiction.

Sufficiency. Is obvious.

Lemma 5. Let $(X, \tau, A)$ be a STS and let $M \in S S(X, A)$. Then $a_{x} \widetilde{\in} C l_{\tau}(M)$ if and only if for all $F \in \tau$ with $a_{x} \widetilde{\in} F$ we have $F \widetilde{\cap} M \neq 0_{A}$.

Proof. Necessity. Suppose that $a_{x} \widetilde{\in} C l_{\tau}(M)$ and suppose to the contrary that there is $F \in \tau$ with $a_{x} \widetilde{\in} F$ we have $F \widetilde{\cap} M=0_{A}$. Since $F \widetilde{\cap} G=0_{A}$, then $M \widetilde{\subseteq} 1_{A}-F$. So, $C l_{\tau}(M) \widetilde{\subseteq} 1_{A}-F$. Since $a_{x} \widetilde{\in} C l_{\tau}(M)$, then $a_{x} \widetilde{\in} 1_{A}-F$, a contradiction.

Sufficiency. Suppose to the contrary that $a_{x} \widetilde{\in}\left(1_{A}-C l_{\tau}(M)\right) \in \tau$. Then by assumption, $\left(1_{A}-C l_{\tau}(M)\right) \widetilde{\cap} M=0_{A}$, a contradiction.

Theorem 14. Let $(X, \tau, A)$ be soft anti-locally countable. Then for all $G \in \tau_{\omega}, C l_{\tau}(G)=C l_{\tau_{\omega}}(G)$.

Proof. Let $(X, \tau, A)$ be soft anti-locally countable and let $G \in \tau_{\omega}$. Clearly that $C l_{\tau_{\omega}}(G) \widetilde{\subseteq} C l_{\tau}(G)$. Conversely, suppose to the contrary that there is $a_{x} \widetilde{\in} C l_{\tau}(G)-C l_{\tau_{\omega}}(G)$. Since $M \in \tau_{\omega}$ such that $a_{x} \widetilde{\in} M$ but $G \widetilde{\cap} M=0_{A}$. Choose $F \in \tau$ and $H \in \operatorname{CSS}(X, A)$ such that $a_{x} \widetilde{\in} F-H \widetilde{\subseteq} M$. Thus, $G \widetilde{\cap}(F-H)=0_{A}$ and hence $G \widetilde{\cap} F \widetilde{\subseteq} H$ which implies that $G \widetilde{\cap} F \in \operatorname{CSS}(X, A)$. Since $a_{x} \widetilde{\in} C l_{\tau}(G)$, then $F \widetilde{\cap} G \neq 0_{A}$. Since $F \widetilde{\cap} G \in \tau_{\omega}-\left\{0_{A}\right\}$ and by Theorem $13\left(X, \tau_{\omega}, A\right)$ is soft anti-locally countable, then $F \widetilde{\cap} G \notin \operatorname{CSS}(X, A)$, a contradiction.

The following example shows that Theorem 14 is no longer true if the assumption of being soft anti-locally countable is omitted:

Example 2. Consider $X=\mathbb{Z}, A=\mathbb{R}, a=2$ and $x=3$ and let $G=a_{x}$. Let $\tau=\left\{0_{A}, 1_{A}, G\right\}$. Then $a_{x} \in \tau$ $\subseteq \tau_{\omega}$, we see that $C l_{\tau}(G)=1_{A}$ but $C l_{\tau_{\omega}}(G)=G \neq 1_{A}$.

The following example shows in Theorem 14 that the assumption ' $G \in \tau_{\omega}$ ' can not be dropped:

Example 3. Consider $\left(\mathbb{R}, \tau\left(\Im_{u}\right), \mathbb{Z}\right)$ where $\Im_{u}$ is the usual topology on $\mathbb{R}$. Let $G \in S S(\mathbb{R}, \mathbb{Z})$ where $G(x)=\mathbb{Q}-\{x\}$ for all $x \in \mathbb{Z}$. Since $G \in C S S(\mathbb{R}, \mathbb{Z})$, then by Theorem $2(d) G$ is soft closed in $\left(X, \tau_{\omega}, A\right)$, and so $\mathrm{Cl}_{\left(\tau\left(\Im_{u}\right)\right)_{\omega}}(G)=G$. On the other hand, it is not difficult to check that $C l_{\tau\left(\Im_{u}\right)}(G)=1_{A}$. 
Notation 2. Let $X$ be a non empty set, $A$ be a set of parameters, $Y$ be a non empty subset of $X$. If $F \in S S(X, A)$, then $F_{Y} \in S S(Y, A)$ is defined by $F_{Y}(a)=F(a) \cap Y$.

Definition 21. [57] Let $(X, \tau, A)$ be a STS and $Y$ be a non empty subset of $X$. Then $\tau_{Y}=\left\{F_{Y}: F \in \tau\right\}$ is said to be the soft relative topology on $Y$ and $\left(Y, \tau_{Y}, A\right)$ is called a soft subspace of $(X, \tau, A)$.

Lemma 6. Let $(X, \tau, A)$ be a STS and $Y$ be a non empty subset of $X$. If $\mathcal{B}$ is a soft base for $\tau$, then $\left\{G_{Y}: G \in \mathcal{B}\right\}$ is a soft base for $\tau_{Y}$.

Proof. Straightforward.

Theorem 15. Let $(X, \tau, A)$ be a STS and $Y$ be a non empty subset of $X$. Then $\left(\tau_{Y}\right)_{\omega}=\left(\tau_{\omega}\right)_{Y}$.

Proof. To see that $\left(\tau_{Y}\right)_{\omega} \subseteq\left(\tau_{\omega}\right)_{Y}$, by Theorem 2 (c) it is sufficient to show that $\left(\tau_{Y}\right)_{c} \subseteq\left(\tau_{\omega}\right)_{Y}$. Let $G \in\left(\tau_{Y}\right)_{c}$. Then there are $F \in \tau$ and $H \in \operatorname{CSS}(Y, A)$ such that $G=F_{Y}-H$. Let $M \in \operatorname{CSS}(X, A)$ where $M(a)=H(a)$ for all $a \in A$. Then $G=F_{Y}-M_{Y}=(F-M)_{Y}$. Since $F-M \in \tau_{\omega}$, then $G \in\left(\tau_{\omega}\right)_{Y}$. To show that $\left(\tau_{\omega}\right)_{Y} \subseteq\left(\tau_{Y}\right)_{\omega}$, by Lemma 6 and Theorem 2 (c) it is sufficient to show that $\left\{(F-H)_{Y}: F \in \tau\right.$ and $\left.H \in \operatorname{CSS}(X, A)\right\} \subseteq\left(\tau_{Y}\right)_{\omega}$. Let $F \in \tau$ and $H \in \operatorname{CSS}(X, A)$. Then $(F-H)_{Y}=$ $F_{Y}-H_{Y}$ with $F_{Y} \in \tau_{Y}$ and $H_{Y} \in \operatorname{CSS}(Y, A)$. Hence, $(F-H)_{Y} \in\left(\tau_{Y}\right)_{\omega}$.

\section{Strongly Soft Omega Open Sets}

In this section, we introduce and investigate strongly soft $\omega$-open sets as a class of soft sets which lies between the class of soft open sets and the class of soft $\omega$-open sets.

Definition 22. Let $G \in S S(X, A)$. The set $\{a \in A: G(a) \neq \varnothing\}$ is called the support of $G$ and is denoted $\operatorname{Supp}(G)$.

Definition 23. Let $G \in S S(X, A)$. Then $G$ is called a strongly countable soft set if $G$ is a countable soft set and $\operatorname{Supp}(G)$ is countable. The set of all countable soft sets from $\operatorname{SS}(X, A)$ will be denoted by $\operatorname{SCSS}(X, A)$.

Proposition 10. Let $G \in S S(X, A)$. Then $G \in \operatorname{SCSS}(X, A)$ if and only if the set $\left\{a_{x}: a_{x} \widetilde{\in} G\right\}$ is countable.

Proof. Straightforward.

Proposition 11. Let $X$ be an initial universe and $A$ be a set of parameters. Then $\operatorname{SCSS}(X, A) \subseteq \operatorname{CSS}(X, A)$.

Proof. Straightforward.

The following example shows in Proposition 11 that $\operatorname{SCSS}(X, A) \neq \operatorname{CSS}(X, A)$ in general:

Example 4. Consider $F \in S S(\mathbb{N}, \mathbb{R})$ with $F(a)=\{1\}$ for all $a \in \mathbb{R}$. Then $F \in \operatorname{CSS}(X, A)-\operatorname{SCSS}(X, A)$.

Theorem 16. Let $X$ be an initial universe and $A$ be a set of parameters. Then $\operatorname{SCSS}(X, A)=\operatorname{CSS}(X, A)$ if and only if $A$ is countable.

Proof. Necessity. Suppose that $\operatorname{SCSS}(X, A)=\operatorname{CSS}(X, A)$. Pick $x \in X$. Let $F \in \operatorname{SS}(X, A)$ where $F(a)=\{x\}$ for all $a \in A$. Then $F \in \operatorname{CSS}(X, A)$. Since $\operatorname{SCSS}(X, A)=\operatorname{CSS}(X, A)$, then $F \in \operatorname{SCSS}(X, A)$. So $\operatorname{Supp}(F)$ is countable. Since $\operatorname{Supp}(F)=A$, then $A$ is countable.

Sufficiency. Suppose that $A$ is countable. By Proposition 11, $\operatorname{SCSS}(X, A) \subseteq \operatorname{CSS}(X, A)$. Let $F \in$ $\operatorname{CSS}(X, A)$. Then $\operatorname{Supp}(F) \subseteq A$. Since $A$ is countable, then $\operatorname{Supp}(F)$. It follows that $F \in \operatorname{CSS}(X, A)$. Therefore, $\operatorname{CSS}(X, A) \subseteq \operatorname{SCSS}(X, A)$ and hence $\operatorname{SCSS}(X, A)=\operatorname{CSS}(X, A)$. 
Definition 24. Let $(X, \tau, A)$ be a STS and let $G \in S S(X, A)$. Then $G$ is called a strongly soft $\omega$-open set if for all $a_{x} \widetilde{\in} G$, there exist $F \in \tau$ and $H \in \operatorname{SCSS}(X, A)$ such that $a_{x} \widetilde{\in} F-H \widetilde{\subseteq} G$. The collection of all strongly soft $\omega$-open sets in $(X, \tau, A)$ will be denoted by $\tau_{\text {sw }}$.

Theorem 17. Let $(X, \tau, A)$ be a STS and let $G \in S S(X, A)$. Then $G$ is strongly soft $\omega$-open if and only if for every $a_{x} \widetilde{\in} G$ there exist $F \in \tau$ such that $a_{x} \tilde{\in} F$ and $F-G \in \operatorname{SCSS}(X, A)$.

Proof. Necessity. Suppose that $G$ is soft $\omega$-open. Let $a_{x} \widetilde{\in} G$, then there exist $F \in \tau$ and $H \in \operatorname{SCSS}(X, A)$ such that $a_{x} \widetilde{\in} F-H \widetilde{\subseteq} G$. Thus $a_{x} \widetilde{\in} F \in \tau$. Since $F-H \widetilde{\subseteq} G$, then $F-G \widetilde{\subseteq} H$ and so $F-G \in$ $\operatorname{SCSS}(X, A)$.

Sufficiency. Suppose that for every $a_{x} \widetilde{\in} G$ there exist $F \in \tau$ such that $a_{x} \widetilde{\in} F$ and $F-G \in$ $\operatorname{SCSS}(X, A)$. Let $a_{x} \widetilde{\in} G$, then there exist $F \in \tau$ such that $a_{x} \widetilde{\in} F$ and $F-G \in \operatorname{SCSS}(X, A)$. Put $H=$ $F-G$. Then $H \in \operatorname{SCSS}(X, A)$ and $F-H \widetilde{\subseteq} G$. It follows that $G$ is strongly soft $\omega$-open.

Notation 3. For a STS $(X, \tau, A)$, denote the collection $\{F-H: F \in \tau$ and $H \in \operatorname{SCSS}(X, A)\}$ by $\tau_{s c}$.

Theorem 18. Let $(X, \tau, A)$ be a STS. Then
(a) $\tau \subseteq \tau_{s c} \subseteq \tau_{s \omega}$.
(b) $\left(X, \tau_{s \omega}, A\right)$ is a STS.
(c) $\tau_{s c}$ is a base for $\tau_{s \omega}$.
(d) Strongly countable soft sets are soft closed in $\left(X, \tau_{s \omega}, A\right)$.
(e) $\tau_{s c} \subseteq \tau_{c}$.
(f) $\tau_{s \omega} \subseteq \tau_{\omega}$.

Proof. (a) Since $0_{A} \in \operatorname{SCSS}(X, A)$, then $\tau=\left\{F-0_{A}: F \in \tau\right\} \subseteq \tau_{s c}$. On the other hand, $\tau_{s c} \subseteq \tau_{s \omega}$ is obvious.

(b) (1) Since $0_{A}, 1_{A} \in \tau$, then by (a) $0_{A}, 1_{A} \in \tau_{s \omega}$.

(2) Let $F, G \in \tau_{s \omega}$ and let $a_{x} \widetilde{\in} F \widetilde{\cap} G$. Then $a_{x} \widetilde{\in} F$ and $a_{x} \widetilde{\in} G$. Then by Theorem 17 , there exist $H, W \in \tau$ such that $a_{x} \widetilde{\in} H \widetilde{\cap} W \in \tau$ and $H-F, W-G \in \operatorname{SCSS}(X, A)$. It is not difficult to check that

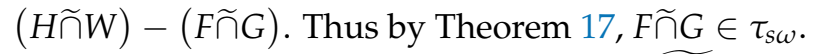

(3) Let $\left\{G_{\alpha}: \alpha \in \Delta\right\} \subseteq \tau_{s \omega}$ and let $a_{x} \widetilde{\in} \widetilde{\bigcup}_{\alpha \in \Delta} G_{\alpha}$. Then there is $\beta \in \Delta$ such that $a_{x} \widetilde{\in} G_{\beta}$. So, there exist $F \in \tau$ and $H \in \operatorname{SCSS}(X, A)$ such that $a_{x} \widetilde{\in} F-H \widetilde{\subseteq} G_{\beta} \widetilde{\subseteq} \widetilde{\bigcup}_{\alpha \in \Delta} G_{\alpha}$. Therefore, $\widetilde{\bigcup}_{\alpha \in \Delta} G_{\alpha} \in \tau_{s \omega}$.

(c) Obvious.

(d) Follows because by (a), $\tau_{s c} \subseteq \tau_{s \omega}$.

(e) Straightforward.

(f) Follows from (c), (e) and Theorem 2 (b).

The following example shows that the inclusion in Theorem 18 (f) cannot be replaced by equality, in general.

Example 5. Let $X$ be a set which contains at least two distinct points, $A$ be an uncountable set of parameters, and $\tau=\left\{1_{A}, 0_{A}\right\}$. Pick $x, y \in X$ with $x \neq y$ and choose $b \in A$. Consider the soft set defined by $F(a)=\{x\}$ for all $a \in A$. Then $1_{A}-F \in \tau_{c} \subseteq \tau_{\omega}$ with $b_{y} \widetilde{\in} 1_{A}-F$. On the other hand, if $1_{A}-F \in \tau_{s \omega}$, then there are $G \in \tau$ and $H \in \operatorname{SCSS}(X, A)$ such that $b_{y} \widetilde{\in} G-H \widetilde{\subseteq} 1_{A}-F$. So $G=1_{A}$ and $1_{A}-H \widetilde{\subseteq} 1_{A}-F$ which implies that $F \widetilde{\widetilde{C}} H$. Since $\operatorname{Supp}(F)=A$, then $\operatorname{Supp}(H)=A$ is uncountable, but $\operatorname{Supp}(H)$ is countable. Therefore, $1_{A}-F \notin \tau_{s \omega}$.

Theorem 19. Let $(X, \tau, A)$ be a STS.

(a) If $A$ is countable, then $\tau_{\omega}=\tau_{s \omega}$.

(b) If $\operatorname{coc}(X, A) \subseteq \tau$, then $\tau_{\omega}=\tau_{s \omega}$. 
Proof. (a) Suppose that $A$ is countable. Then by Theorem16, $\operatorname{SCSS}(X, A)=\operatorname{CSS}(X, A)$. So $\tau_{s c}=\tau_{c}$ and by Theorems 2 (c) and 18 (c) it follows that $\tau_{\omega}=\tau_{s \omega}$.

(b) Suppose that $\operatorname{coc}(X, A) \subseteq \tau$. Then by Theorem $4, \tau_{\omega}=\tau$. So by Theorem 18 (a) $\tau_{\omega}=\tau \subseteq \tau_{s \omega}$. Also, by Theorem 18 (f) we have $\tau_{s \omega} \subseteq \tau_{\omega}$.

The following example shows that the implication in Theorem 19 (a) is not reversible, in general:

Example 6. Let $X$ be an initial universe, $A$ be an uncountable set of parameters and let $\tau=\operatorname{coc}(X, A)$. Then by Theorem $19(b), \tau_{\omega}=\tau_{s \omega}$.

The following example shows that the implication in Theorem 19 (b) is not reversible, in general:

Example 7. Let $X=\mathbb{R}, A=\mathbb{N}$ and $\tau=\left\{1_{A}, 0_{A}\right\}$. Then by Theorem 19 (a) we have $\tau_{\omega}=\tau_{s \omega}$. On the other hand, it is clear that $\operatorname{coc}(X, A)$ is not a subset of $\tau$.

Theorem 20. Let $X$ be an initial universe, $A$ be a set of parameters and let $\tau=\left\{0_{A}\right\} \cup$ $\left\{1_{A}-H: H \in \operatorname{SCSS}(X, A)\right\}$. Then $(X, \tau, A)$ is a STS.

Proof. (1) By definition of $\tau, 0_{A} \in \tau$. Since $0_{A} \in \operatorname{SCSS}(X, A)$, then $1_{A}=\left(1_{A}-0_{A}\right) \in \tau$.

(2) Let $1_{A}-H, 1_{A}-K \in \tau-\left\{0_{A}\right\}$. Then $H \widetilde{\cup} K \in \operatorname{SCSS}(X, A)$ and $\left(1_{A}-H\right) \widetilde{\cap}\left(1_{A}-K\right)=$ $\left(1_{A}-(H \widetilde{\cup} K)\right) \in \tau$.

(3) Let $\left\{1_{A}-H_{\alpha}: \alpha \in \Delta\right\} \subseteq \tau-\left\{0_{A}\right\}$. Then $\widetilde{\cap}\left\{H_{\alpha}: \alpha \in \Delta\right\} \in \operatorname{SCSS}(X, A)$ and so $\widetilde{\cup}\left\{1_{A}-H_{\alpha}: \alpha \in \Delta\right\}=1_{A}-\widetilde{\cap}\left\{H_{\alpha}: \alpha \in \Delta\right\} \in \tau$.

Definition 25. Let $(X, \tau, A)$ be a STS. Then $\left\{0_{A}\right\} \cup\left\{1_{A}-H: H \in \operatorname{CSS}(X, A)\right\}$ will be called the strongly cocountable soft topology and will be denoted by $\operatorname{scoc}(X, A)$.

Proposition 12. For any $\operatorname{STS}(X, \tau, A), \operatorname{scoc}(X, A) \subseteq \tau_{s c}$.

Proof. Obvious.

Theorem 21. For any STS $(X, \tau, A)$, the following are equivalent:
(a) $\operatorname{scoc}(X, A) \subseteq \tau$.
(b) $\tau=\tau_{s c}$.
(c) $\tau=\tau_{s \omega}$.

Proof. (a) $\Longrightarrow$ (b): Suppose that $\operatorname{scoc}(X, A) \subseteq \tau$. We need only to show that $\tau_{s c} \subseteq \tau$. Let $G \in \tau$ and $H \in \operatorname{SCSS}(X, A)$. Then $G-H=G \widetilde{\cap}\left(1_{A}-H\right)$. Since $\operatorname{scoc}(X, A) \subseteq \tau$, then $1_{A}-H \in \tau$ and so $G-H \in \tau$. It follows that $\tau_{s c} \subseteq \tau$.

(b) $\Longrightarrow$ (c): Suppose that $\tau=\tau_{s c}$. Then $\tau_{s c}$ is a soft topology. By Theorem 18 (c), it follows that $\tau_{s c}=\tau_{s \omega}$ and hence $\tau=\tau_{s \omega}$.

(c) $\Longrightarrow$ (a): Suppose that $\tau=\tau_{s \omega}$. Then by Proposition 12 and Theorem 18 (a), we have $\operatorname{scoc}(X, A) \subseteq \tau_{s c} \subseteq \tau_{s \omega}=\tau$.

Corollary 7. Let $X$ be an initial universe and $A$ be a set of parameters. Then $(\operatorname{scoc}(X, A))_{s \omega}=\operatorname{scoc}(X, A)$.

Theorem 22. For any STS $(X, \tau, A)$ we have $\tau_{s \omega}=\left(\tau_{s \omega}\right)_{s \omega}$.

Proof. By Proposition 4.17 and Theorem 4.10 (a), we have $\operatorname{scoc}(X, A) \subseteq \tau_{s c} \subseteq \tau_{s \omega}$. Then by Theorem 21 , it follows that $\tau_{s \omega}=\left(\tau_{s \omega}\right)_{s \omega}$.

Theorem 23. For any STS $(X, \tau, A)$ we have $\tau_{\omega}=\left(\tau_{s \omega}\right)_{\omega}=\left(\tau_{\omega}\right)_{s \omega}$. 
Proof. By Theorem 18(a) and (f) we have $\tau \subseteq \tau_{s \omega}, \tau_{\omega} \subseteq\left(\tau_{\omega}\right)_{s \omega}, \tau_{s \omega} \subseteq \tau_{\omega}$ and $\left(\tau_{\omega}\right)_{s \omega} \subseteq\left(\tau_{\omega}\right)_{\omega}=\tau_{\omega}$. Thus, we have $\tau_{\omega} \subseteq\left(\tau_{s \omega}\right)_{\omega}, \tau_{\omega} \subseteq\left(\tau_{\omega}\right)_{s \omega^{\prime}}\left(\tau_{s \omega}\right)_{\omega} \subseteq\left(\tau_{\omega}\right)_{\omega}=\tau_{\omega}$ and $\left(\tau_{\omega}\right)_{s \omega} \subseteq \tau_{\omega}$. Therefore, $\tau_{\omega}=\left(\tau_{s \omega}\right)_{\omega}=\left(\tau_{\omega}\right)_{s \omega}$.

Theorem 24. Let $(X, \tau, A)$ and $(X, \sigma, A)$ be two STS's. If $\tau \cup \operatorname{scoc}(X, A) \subseteq \sigma$, then $\tau_{s c} \subseteq \sigma$.

Proof. Let $F-H \in \tau_{s c}$, where $F \in \tau$ and $H \in \operatorname{SCSS}(X, A)$. Since $F \in \tau, 1_{A}-H \in \operatorname{scoc}(X, A)$ and $\tau \cup \operatorname{scoc}(X, A) \subseteq \sigma$, then $F, 1_{A}-H \in \sigma$ and so $F \widetilde{\cap}\left(1_{A}-H\right)=F-H \in \sigma$.

Corollary 8. Let $(X, \tau, A)$ and $(X, \sigma, A)$ be two STS's. If $\tau \cup \operatorname{scoc}(X, A) \subseteq \sigma$, then $\tau_{s \omega} \subseteq \sigma$.

Proof. Follows from Theorem 24 and Theorem 18 (c).

Theorem 25. Let $(X, \tau, A)$ be a STS. Then for all $a \in A,\left(\tau_{a}\right)_{\omega}=\left(\tau_{s \omega}\right)_{a}$.

Proof. Let $a \in A$. To show that $\left(\tau_{a}\right)_{\omega} \subseteq\left(\tau_{s \omega}\right)_{a}$, it is sufficient to see that $U-C \in\left(\tau_{s \omega}\right)_{a}$ for all $U \in \tau_{a}$ and a countable subset $C \subseteq X$. Let $U \in \tau_{a}$ and let $C$ be a countable subset of $X$. Since $U \in \tau_{a}$, then there is $F \in \tau$ such $F(a)=U$. Let $H=a_{C}$, then $H \in \operatorname{SCSS}(X, A)$. Since we have $F-H \in$ $\tau_{s \omega}$, then $(F-H)(a)=F(a)-H(a)=U-C \in\left(\tau_{s \omega}\right)_{a}$. To show that $\left(\tau_{s \omega}\right)_{a} \subseteq\left(\tau_{a}\right)_{\omega}$, by Theorem 2 (c) and Lemma 1 it is sufficient to show that $\{(F-H)(a): F \in \tau$ and $H \in \operatorname{SCSS}(X, A)\} \subseteq\left(\tau_{a}\right)_{\omega}$. Let $F \in \tau$ and $H \in \operatorname{SCSS}(X, A)$, then $(F-H)(a)=F(a)-H(a)$ with $F(a) \in \tau_{a}$ and $H(a)$ is a countable subset of $X$ which implies that $(F-H)(a) \in\left(\tau_{a}\right)_{\omega}$.

Corollary 9. Let $(X, \tau, A)$ be a STS. If $G \in \tau_{s \omega}$, then for all $a \in A$ we have $G(a) \in\left(\tau_{a}\right)_{\omega}$.

Proof. Let $G \in \tau_{s \omega}$ and let $a \in A$. Then $G(a) \in\left(\tau_{s \omega}\right)_{a}$ and by Theorem 25 we have $G(a) \in\left(\tau_{a}\right)_{\omega}$.

Corollary 10. Let $(X, \tau, A)$ be a STS. Then for all $a \in A,\left(\tau_{\omega}\right)_{a}=\left(\tau_{s \omega}\right)_{a}$.

Proof. Follows from Theorems 7 and 25.

Theorem 26. Let $X$ be an initial universe and let $A$ be a set of parameters. Let $\left\{\Im_{a}: a \in A\right\}$ be an indexed family of topologies on $X$. Then $\left(\bigoplus_{a \in A} \Im_{a}\right)_{s \omega}=\bigoplus_{a \in A}\left(\Im_{a}\right)_{\omega}$.

Proof. To show that $\left(\bigoplus_{a \in A} \Im_{a}\right)_{s \omega} \subseteq \underset{a \in A}{\bigoplus}\left(\Im_{a}\right)_{\omega}$, by Theorem 18 (c) it is sufficient to show that $\left(\bigoplus_{a \in A} \Im_{a}\right)_{s c} \subseteq \underset{a \in A}{\bigoplus_{a}}\left(\Im_{a}\right)_{\omega}$. Let $F \in \bigoplus_{a \in A} \Im_{a}$ and $H \in \operatorname{SCSS}(X, A)$. Then for every $a \in A$, $F(a) \in \Im_{a}$ and $H(a)$ is a countable subset of $X$ and so $(F-H)(a)=F(a)-H(a) \in\left(\Im_{a}\right)_{\omega}$. Thus, $F-H \in \bigoplus_{a \in A}\left(\Im_{a}\right)_{\omega}$. For every $a \in A$, let $\left\{U-C: U \in \Im_{a}\right.$ and $C$ is a countable subset of $\left.X\right\}$ is a base for $\left(\Im_{a}\right)_{\omega}$, so by Lemma $2\left\{a_{U-C}: a \in A, U \in \Im_{a}\right.$ and $C$ is a countable subset of $\left.X\right\}$ is a soft base for $\bigoplus_{a \in A}\left(\Im_{a}\right)_{\omega}$. Thus, to show that $\bigoplus_{a \in A}\left(\Im_{a}\right)_{\omega} \subseteq\left(\bigoplus_{a \in A} \Im_{a}\right)_{\omega}$ it is sufficient to show that $\left\{a_{U-C}: a \in A, U \in \Im_{a}\right.$ and $C$ is a countable subset of $\left.X\right\}{ }^{\omega} \subseteq$ $\left(\bigoplus_{a \in A} \Im_{a}\right)_{s \omega}$. Note that $\left\{a_{U-C}: a \in A, U \in \Im_{a}\right.$ and $C$ is a countable subset of $\left.X\right\}=$ $\left\{a_{U}-a_{C}: a \in A, U \in \Im_{a}\right.$ and $C$ is a countable subset of $\left.X\right\}$, which ends the proof.

Corollary 11. Let $X$ be an initial universe and let $A$ be a set of parameters. Let $\left\{\Im_{a}: a \in A\right\}$ be an indexed family of topologies on $X$. Then $\left(\bigoplus_{a \in A} \Im_{a}\right)_{s \omega}=\left(\bigoplus_{a \in A} \Im_{a}\right)_{\omega}$. 
Proof. Follows from Theorems 8 and 26.

Corollary 12. If $(X, \Im)$ is a topological space and $A$ is any set of parameters, then $(\tau(\Im))_{s \omega}=\tau\left(\Im_{\omega}\right)$.

Proof. For each $a \in A$, set $\Im_{a}=\Im$. Then $\tau(\Im)=\bigoplus_{a \in A} \Im_{a}$ and by Theorem 26,

$$
\begin{aligned}
(\tau(\Im))_{s \omega} & =\left(\bigoplus_{a \in A} \Im_{a}\right)_{s \omega} \\
& =\bigoplus_{a \in A}\left(\Im_{a}\right)_{\omega} \\
& =\tau\left(\Im_{\omega}\right) .
\end{aligned}
$$

Corollary 13. If $(X, \Im)$ is a topological space and $A$ is any set of parameters, then $(\tau(\Im))_{s \omega}=(\tau(\Im))_{\omega}$.

Proof. Follows from Corollaries 4 and 11.

Theorem 27. If $(X, \tau, A)$ is soft $T_{1}$ and soft $p$-space, then $\tau=\tau_{\omega}$.

Proof. Follows from Theorems 9, 18 (a) and 18 (f).

Definition 26. A STS $(X, \tau, A)$ is called strongly soft locally countable if for $a_{x} \in S P(X, A)$ there exists $G \in \operatorname{SCSS}(X, A) \cap \tau$ such that $a_{x} \tilde{\in} G$.

Theorem 28. A STS $(X, \tau, A)$ is strongly soft locally countable if and only if $\operatorname{SCSS}(X, A) \cap \tau$ is a soft base for $(X, \tau, A)$.

Proof. Necessity. Suppose that $(X, \tau, A)$ is strongly soft locally countable. Let $F \in \tau-\left\{0_{A}\right\}$ and let $a_{x} \widetilde{\in} F$. Since $(X, \tau, A)$ is strongly soft locally countable, then there is $G \in \operatorname{SCSS}(X, A) \cap \tau$ such that $a_{x} \widetilde{\in} G$. Then we have $G \widetilde{\cap} F \in \operatorname{SCSS}(X, A) \cap \tau$ with $a_{x} \widetilde{\in} G \widetilde{\cap} F \widetilde{\subseteq} F$. This shows that $\operatorname{SCSS}(X, A) \cap \tau$ is a soft base for $(X, \tau, A)$.

Sufficiency. Suppose that $\operatorname{SCSS}(X, A) \cap \tau$ is a soft base for $(X, \tau, A)$. Let $a_{x} \in \operatorname{SP}(X, A)$. Then $a_{x} \widetilde{\in} 1_{A}$ and so there is $G \in \operatorname{SCSS}(X, A) \cap \tau$ such that $a_{x} \widetilde{\in} G \widetilde{\widetilde{C}} 1_{A}$. This shows that $(X, \tau, A)$ is strongly soft locally countable.

Theorem 29. A STS $(X, \tau, A)$ is strongly soft locally countable if and only if $S P(X, A) \subseteq \tau_{s c}$.

Proof. Necessity. Suppose that $(X, \tau, A)$ is strongly soft locally countable. Let $a_{x} \in S P(X, A)$, then by strong soft local countability of $(X, \tau, A)$, there exists $G \in \operatorname{SCSS}(X, A)$ such that $a_{x} \tilde{\in} G$. Then $G-a_{x} \in$ $\operatorname{SCSS}(X, A)$ and so, $a_{x}=G-\left(G-a_{x}\right) \in \tau_{s c}$.

Sufficiency. Suppose that $\operatorname{SP}(X, A) \subseteq \tau_{s c}$. Let $a_{x} \in S P(X, A)$. Then $a_{x} \in \tau_{s c}$ and so there is $F \in \tau$ and $H \in \operatorname{SCSS}(X, A)$ such that $a_{x}=F-H$. Then $F \in \operatorname{SCSS}(X, A)$ with $a_{x} \tilde{\in} F$. It follows that $(X, \tau, A)$ is strongly soft locally countable.

Corollary 14. A STS $(X, \tau, A)$ is strongly soft locally countable if and only if $\left(X, \tau_{s \omega}, A\right)$ is a discrete STS.

Corollary 15. If $(X, \tau, A)$ is a STS with $X$ and $A$ are countable, then $\left(X, \tau_{s \omega}, A\right)$ is a discrete STS.

Theorem 30. For any STS $(X, \tau, A),\left(X, \tau_{s \omega}, A\right)$ is soft $T_{1}$. 
Proof. Follows from Theorem 18 (d) and Lemma 4.

Theorem 31. If $(X, \tau, A)$ is a soft $T_{2}$ STS, then $\left(X, \tau_{s \omega}, A\right)$ is soft $T_{2}$.

Proof. Let $a_{x}, a_{y} \in S P(X, A)$ with $x \neq y$. Since $(X, \tau, A)$ is soft $T_{2}$, then there exist $G, F \in \tau$ such that $a_{x} \widetilde{\in} G, a_{y} \widetilde{\in} F$ and $G \widetilde{\cap} F=0_{A}$. By Theorem $4(\mathrm{a}), \tau \subseteq \tau_{s \omega}$ and so $G, F \in \tau_{s \omega}$ which ends the proof that $\left(X, \tau_{s \omega}, A\right)$ is soft $T_{2}$.

The following example shows that the converse of Theorem 31 need not to be true in general:

Example 8. Let $X=\mathbb{N}, A=\mathbb{Z}$ and $\tau=\left\{1_{A}, 0_{A}\right\}$. By Corollary $15,\left(X, \tau_{s \omega}, A\right)$ is a discrete STS. Thus, $\left(X, \tau_{s \omega}, A\right)$ is soft $T_{2}$. On the other hand, it is clear that $(X, \tau, A)$ is not soft $T_{2}$.

Definition 27. A STS $(X, \tau, A)$ is called strongly soft anti-locally countable if for every $F \in \tau-\left\{0_{A}\right\}$, $F \notin \operatorname{SCSS}(X, A)$.

Theorem 32. A STS $(X, \tau, A)$ is soft anti-locally countable if and only if $\left(X, \tau_{s} \omega, A\right)$ is strongly soft anti-locally countable.

Proof. Necessity. Suppose to the contrary that $(X, \tau, A)$ is soft anti-locally countable and there is $G \in \tau_{s \omega}-\left\{0_{A}\right\}$ with $G \in \operatorname{SCSS}(X, A)$. Choose $a_{x} \widetilde{\in} G$. There are $F \in \tau$ and $H \in \operatorname{SCSS}(X, A)$ such that $a_{x} \widetilde{\in} F-H \widetilde{\subseteq} G$, and so $F \widetilde{\subseteq} H \widetilde{\cup} G$ which implies that $F \in S C S S(X, A)$. Since $F \in \tau-\left\{0_{A}\right\}$ and $(X, \tau, A)$ is strongly soft anti-locally countable, then we have a contradiction.

Sufficiency. Is obvious.

Theorem 33. Let $(X, \tau, A)$ be strongly soft anti-locally countable. Then for all $G \in \tau_{s \omega}, C l_{\tau}(G)=C l_{\tau_{s \omega}}(G)$.

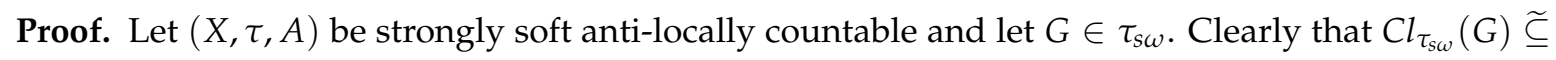
$C l_{\tau}(G)$. Conversely, suppose to the contrary that there is $a_{x} \widetilde{\in} C l_{\tau}(G)-C l_{\tau_{s \omega}}(G)$. There is $M \in \tau_{s \omega}$ such that $a_{x} \widetilde{\in} M$ but $G \widetilde{\cap} M=0_{A}$. Choose $F \in \tau$ and $H \in \operatorname{SCSS}(X, A)$ such that $a_{x} \widetilde{\in} F-H \widetilde{\subseteq} M$. Thus, $G \widetilde{\cap}(F-H)=0_{A}$ and hence $G \widetilde{\cap} F \widetilde{\subseteq} H$ which implies that $G \widetilde{\cap} F \in \operatorname{SCSS}(X, A)$. Since $a_{x} \widetilde{\in} C l_{\tau}(G)$, then $F \widetilde{\cap} G \neq 0_{A}$. Since $F \widetilde{\cap} G \in \tau_{s \omega}-\left\{0_{A}\right\}$ and by Theorem $32\left(X, \tau_{s \omega}, A\right)$ is strongly soft anti-locally countable, then $F \widetilde{\cap} G \notin S C S S(X, A)$, a contradiction.

The following example shows that Theorem 33 is no longer true if the assumption of being strongly soft anti-locally countable is omitted:

Example 9. Consider $X=\mathbb{Z}, A=\mathbb{N}, a=2$ and $x=3$ and let $G=a_{x}$. Let $\tau=\left\{0_{A}, 1_{A}, G\right\}$. Then $a_{x} \in \tau$ $\subseteq \tau_{s \omega}$, we see that $C l_{\tau}(G)=1_{A}$ but $C l_{\tau_{s}}(G)=G \neq 1_{A}$.

The following example shows in Theorem 33 that the assumption ' $G \in \tau_{s \omega}$ ' can not be dropped:

Example 10. Consider $\left(\mathbb{R}, \tau\left(\Im_{u}\right), \mathbb{Z}\right)$ where $\Im_{u}$ is the usual topology on $\mathbb{R}$. Let $G \in S S(\mathbb{R}, \mathbb{Z})$ where $G(x)=\mathbb{Q}-\{x\}$ for all $x \in \mathbb{Z}$. Since $G \in S C S S(\mathbb{R}, \mathbb{Z})$, then by Theorem $18(d) G$ is soft closed in $\left(X, \tau_{s \omega}, A\right)$, and so $C l_{\left(\tau\left(\Im_{u}\right)\right)_{s \omega}}(G)=G$. On the other hand, it is not difficult to check that $C l_{\tau\left(\Im_{u}\right)}(G)=1_{A}$.

Theorem 34. Let $(X, \tau, A)$ be a STS and $Y$ be a non empty subset of $X$. Then $\left(\tau_{Y}\right)_{s \omega}=\left(\tau_{s \omega}\right)_{Y}$.

Proof. To see that $\left(\tau_{Y}\right)_{s \omega} \subseteq\left(\tau_{s \omega}\right)_{Y}$, by Theorem 18 (c) it is sufficient to show that $\left(\tau_{Y}\right)_{s c} \subseteq\left(\tau_{s \omega}\right)_{Y}$. Let $G \in\left(\tau_{Y}\right)_{S c}$. Then there are $F \in \tau$ and $H \in S C S S(Y, A)$ such that $G=F_{Y}-H$. Let $M \in$ $\operatorname{SCSS}(X, A)$ where $M(a)=H(a)$ for all $a \in A$. Then $G=F_{Y}-M_{Y}=(F-M)_{Y}$. Since $F-M \in \tau_{s \omega}$, then $G \in\left(\tau_{s \omega}\right)_{Y}$. To show that $\left(\tau_{s \omega}\right)_{Y} \subseteq\left(\tau_{Y}\right)_{s \omega}$, by Lemma 6 and Theorem 18 (c) it is sufficient 
to show that $\left\{(F-H)_{Y}: F \in \tau\right.$ and $\left.H \in \operatorname{CSS}(X, A)\right\} \subseteq\left(\tau_{Y}\right)_{\omega}$. Let $F \in \tau$ and $H \in \operatorname{SCSS}(X, A)$. Then $(F-H)_{Y}=F_{Y}-H_{Y}$ with $F_{Y} \in \tau_{Y}$ and $H_{Y} \in S C S S(Y, A)$. Hence, $(F-H)_{Y} \in\left(\tau_{Y}\right)_{s \omega}$.

\section{Soft Lindelof STS's and Soft Sw-Open Sets}

In this section, we use strongly soft omega open sets to characterize soft Lindelof STS's.

Definition 28. [58] Let $(X, \tau, A)$ be a STS and let $\sigma \subseteq S S(X, \tau)$.

(1) $\sigma$ is called a soft open cover of $(X, \tau, A)$ if $\sigma \subseteq \tau$ and $\widetilde{U}\{F: F \in \sigma\}=1_{A}$.

(2) A countable subfamily of a soft open cover $\sigma$ of $(X, \tau, A)$ is called a countable subcover of $\sigma$, if it is also a soft open cover of $(X, \tau, A)$.

(3) $(X, \tau, A)$ is called soft Lindelof if every soft open cover of $(X, \tau, A)$ has a countable subcover.

Lemma 7. Let $(X, \tau, A)$ be a STS and let $\mathcal{B}$ be a soft base of $\tau$. Then $(X, \tau, A)$ is soft Lindelof if and only if every soft open cover $\sigma$ of $(X, \tau, A)$ with $\sigma \subseteq \mathcal{B}$ has a countable subcover.

Proof. Necessity. It is obvious

Sufficiency. Suppose that every soft open cover $\sigma$ of $(X, \tau, A)$ with $\sigma \subseteq \mathcal{B}$ has a countable subcover. Let $\sigma$ be a soft open cover of $(X, \tau, A)$ with $\sigma \subseteq \tau-\left\{0_{A}\right\}$. For every $F \in \sigma$, there is $\mathcal{B}_{F} \subseteq \mathcal{B}$ such that $\widetilde{\cup}\left\{G: G \in \mathcal{B}_{F}\right\}=F$. Let $\mu=\left\{G: G \in \mathcal{B}_{F}, F \in \sigma\right\}$. Then $\widetilde{\cup}\{G: G \in \mu\}=1_{A}$ and by assumption, there is a countable subcover $\rho$ of $\mu$. For each $G \in \rho$, choose $F(G) \in \sigma$ such that $G \subseteq \widetilde{\subseteq} F(G)$. Then $\{F(G): G \in \rho\}$ is a countable subcover of $\sigma$.

Theorem 35. A STS $(X, \tau, A)$ is soft Lindelof if and only if $\left(X, \tau_{s \omega}, A\right)$ is soft Lindelof.

Proof. Necessity. Suppose that $(X, \tau, A)$ is soft Lindelof. By Theorem $18(\mathrm{c}), \tau_{s c}$ is a soft base of $\tau_{s \omega}$, so by Lemma 7 it is sufficient to show that every soft open cover $\sigma$ of $\left(X, \tau_{s \omega}, A\right)$ with $\sigma \subseteq \tau_{s c}$ has a countable subcover. Let $\sigma$ be a soft open cover of $\left(X, \tau_{s \omega}, A\right)$ with $\sigma \subseteq \tau_{s c}$, say $\sigma=\left\{F_{\alpha}-H_{\alpha}: \alpha \in \Delta\right\}$ with $F_{\alpha} \in \tau$ and $H_{\alpha} \in \operatorname{SCSS}(X, \tau)$ for all $\alpha \in \Delta$. Then $\left\{F_{\alpha}: \alpha \in \Delta\right\}$ is a soft open cover of $(X, \tau, A)$. Since $(X, \tau, A)$ is soft Lindelof, then there is a countable subset $\Delta_{1} \subseteq \Delta$ such that $\left\{F_{\alpha}: \alpha \in \Delta_{1}\right\}$ is also a soft open cover of $(X, \tau, A)$. Put $H=\widetilde{\bigcup}\left\{H_{\alpha}: \alpha \in \Delta_{1}\right\}$. Then $H$ is strongly soft countable. For each $a_{x} \widetilde{\in} H$, take $\alpha\left(a_{x}\right) \in \Delta$ such that $a_{x} \widetilde{\in} F_{\alpha\left(a_{x}\right)}-H_{\alpha\left(a_{x}\right)}$. Therefore, $\left\{F_{\alpha}-H_{\alpha}: \alpha \in \Delta_{1}\right\} \cup\left\{F_{\alpha\left(a_{x}\right)}-H_{\alpha\left(a_{x}\right)}: a_{x} \widetilde{\in} H\right\}$ is a countable subcover of $\sigma$. It follows that $\left(X, \tau_{s \omega}, A\right)$ is soft Lindelof.

Sufficiency. It is obvious.

Corollary 16. Let $(X, \tau, A)$ be a STS, where $\tau=\operatorname{scoc}(X, A)$ is soft Lindelof.

Proof. Consider $(X, \sigma, A)$ where $\sigma=\left\{0_{A}, 1_{A}\right\}$. Then $(X, \sigma, A)$ is obviously soft Lindelof. So by Theorem $35,\left(X, \sigma_{s \omega}, A\right)$ is soft Lindelof. Since $\sigma_{s \omega}=\operatorname{scoc}(X, A)$, then $(X, \tau, A)$ is soft Lindelof.

Theorem 36. Let $(X, \tau, A)$ be a STS. If $\left(X, \tau_{\omega}, A\right)$ is soft Lindelof, then $(X, \tau, A)$ is soft Lindelof.

Proof. Follows because by Theorem 2 (a) we have $\tau \subseteq \tau_{\omega}$.

Theorem 37. Let $(X, \tau, A)$ be a STS where $\tau=\operatorname{coc}(X, A)$. Then $(X, \tau, A)$ is soft Lindelof if and only if $A$ is countable.

Proof. Necessity. Suppose that $(X, \tau, A)$ is soft Lindelof and suppose to the contrary that $A$ is uncountable. Pick $x_{\circ} \in X$ and let $H \in \operatorname{CSS}(X, A)$ where $H(a)=\left\{x_{\circ}\right\}$. For each $a \in A$, let $G_{a}=\left(1_{A}-H\right) \widetilde{\cup} a_{x_{0}}$. Then $\left\{G_{a}: a \in A\right\}$ is a soft open cover of $(X, \tau, A)$ which contains no countable subcover. Thus, $(X, \tau, A)$ is not soft Lindelof which is a contradiction. 
Sufficiency. Suppose that $A$ is countable. Consider $(X, \sigma, A)$ where $\sigma=\left\{0_{A}, 1_{A}\right\}$. Then $\sigma_{\omega}=\operatorname{coc}(X, A)$ and $\sigma_{s \omega}=\operatorname{scoc}(X, A)$. So by Theorem 19 (a) $\operatorname{coc}(X, A)=\operatorname{scoc}(X, A)$. Therefore, by Corollary $16(X, \tau, A)$ is soft Lindelof.

The following example shows that the converse of Theorem 36 is not true in general:

Example 11. Let $X$ be a non empty set and let be an uncountable of parameters. Let $\tau=\left\{0_{A}, 1_{A}\right\}$. Then clearly that $(X, \tau, A)$ is soft Lindelof. On the other hand since $\tau_{\omega}=\operatorname{coc}(X, A)$ and $A$ is uncountable, then by Theorem $37\left(X, \tau_{\omega}, A\right)$ is not soft Lindelof.

Theorem 38. Let $X$ be an initial universe and let $A$ be a set of parameters. Let $\left\{\Im_{a}: a \in A\right\}$ be an indexed family of topologies on $X$. Then $\left(X, \bigoplus_{a \in A} \Im_{a}, A\right)$ is soft Lindelof if and only if $A$ is countable and $\left(X, \Im_{a}\right)$ is Lindelof for all $a \in A$.

Proof. Necessity. Suppose that $\left(X, \bigoplus_{a \in A} \Im_{a}, A\right)$ is soft Lindelof. Since $\left\{a_{X}: a \in A\right\}$ is a soft open cover of $\bigoplus_{a \in A} \Im_{a}$, it contains a countable subcover $\left\{a_{X}: a \in A_{1}\right\}$ where $A_{1}$ is a countable subset of $A$. It is not difficult to show that $A_{1}=A$ and hence $A$ is countable. Let $b \in A$. To show that $\left(X, \Im_{b}\right)$ is Lindelof, let $\mathcal{M} \subseteq \Im_{b}$ with $\cup\{M: M \in \mathcal{M}\}$. Let $\sigma=\left\{b_{M}: M \in \mathcal{M}\right\} \cup\left\{a_{X}: a \in A-\{b\}\right\}$. Then $\sigma$ is a soft open cover of the soft Lindelof STS $\left(X, \underset{a \in A}{\bigoplus_{a}} \Im_{a} A\right)$ and so it has a countable subcover $\rho$. It is not difficult to show that there is a countable subfamily $\mathcal{M}_{1}$ of $\mathcal{M}$ such that $\rho=\left\{b_{M}: M \in \mathcal{M}_{1}\right\} \cup$ $\left\{a_{X}: a \in A-\{b\}\right\}$. Therefore, $\mathcal{M}_{1}$ is a countable subcover of $\mathcal{M}$ and hence $\left(X, \Im_{b}\right)$ is Lindelof.

Sufficiency. Suppose that $A$ is countable and $\left(X, \Im_{a}\right)$ is Lindelof for all $a \in A$. Let $\mathcal{B}=$ $\left\{a_{Y}: a \in A\right.$ and $\left.Y \in \Im_{a}\right\}$. By Proposition $7, \mathcal{B}$ is a soft base of $\bigoplus_{a \in A} \Im_{a}$. We apply Lemma 7. Let $\sigma$ be a soft open cover of $\left(X, \underset{a \in A}{\bigoplus_{a}} \Im_{a}, A\right)$ with $\sigma \subseteq \mathcal{B}$. For each $a \in A$, let $\sigma_{a}=\left\{Y \subseteq X: a_{Y} \in \sigma\right\}$. Then for all $a \in A, \sigma_{a}$ is an open cover of the Lindelof topological space $\left(X, \Im_{a}\right)$ and so $\sigma_{a}$ contains a countable subcover $\rho_{a}$. Let $\rho=\left\{a_{Y}: a \in A\right.$ and $\left.a \in \rho_{a}\right\}$. Since $A$ is countable, then $\rho$ is countable. Therefore, $\rho$ is a countable subcover of $\sigma$. It follows that $\left(X, \underset{a \in A}{\bigoplus_{a}} \Im_{a} A\right)$ is soft Lindelof.

Theorem 39. Let $X$ be an initial universe and let $A$ be a set of parameters. Let $\left\{\Im_{a}: a \in A\right\}$ be an indexed family of topologies on $X$. Then the following are equivalent:
(a) $\left(X,\left(\bigoplus_{a \in A} \Im_{a}\right)_{\omega}, A\right)$ is soft Lindelof.
(b) $\left(X, \bigoplus_{a \in A} \Im_{a}, A\right)$ is soft Lindelof.
(c) $\left(X,\left(\bigoplus_{a \in A} \Im_{a}\right)_{s \omega}, A\right)$ is soft Lindelof.

Proof. (a) $\Longrightarrow$ (b): Follows by Theorem 36 .

(b) $\Longrightarrow$ (c): Follows by Theorem 35.

(c) $\Longrightarrow$ (a): Follows by Corollary 11 .

\section{Soft Weakly Lindelof STS's}

In this section, we define and investigate soft weakly Lindelof STS's. In particular, we characterize soft weakly Lindelof sets STS's which are strongly soft anti-locally countable via $s \omega$-open sets.

Definition 29. [59] A STS $(X, \tau, A)$ is called soft separable if there is $F \in \operatorname{SCSS}(X, A)$ such that $1_{A}=$ $\mathrm{Cl}_{\tau}(F)$. 
Definition 30. A STS $(X, \tau, A)$ is called soft weakly Lindelof if every soft open cover $\sigma$ of $(X, \tau, A)$ contains a countable subfamily $\rho$ such that $1_{A}=C l_{\tau}(\widetilde{\cup}\{F: F \in \rho\})$.

Definition 31. [60] A topological space $(X, \Im)$ is called weakly Lindelof if every soft open cover $\mathcal{M}$ of $(X, \Im)$ contains a countable subfamily $\mathcal{M}_{1}$ such that $X=\overline{\bigcup\left\{M: M \in \mathcal{M}_{1}\right\}}$.

Theorem 40. Every soft Lindelof STS is soft weakly Lindelof.

Proof. Let $(X, \tau, A)$ be soft Lindelof and let $\sigma$ be a soft open cover of $(X, \tau, A)$. Since $(X, \tau, A)$ is soft Lindelof, then there is a countable subfamily $\gamma$ of $\sigma$ which is also a soft open cover of $(X, \tau, A)$. Thus, we have

$$
1_{A}=\widetilde{\bigcup}\{F: F \in \gamma\} \widetilde{\subseteq} C l_{\tau}(\widetilde{\bigcup}\{F: F \in \gamma\})
$$

and hence $1_{A}=C l_{\tau}(\widetilde{\bigcup}\{F: F \in \gamma\})$. This shows that $(X, \tau, A)$ is soft weakly Lindelof.

Theorem 41. Every soft separable STS is soft weakly Lindelof.

Proof. Let $(X, \tau, A)$ be soft separable. Let $\sigma$ be a soft open cover of $(X, \tau, A)$. Since $(X, \tau, A)$ is soft separable, then there is a $G \in \operatorname{SCSS}(X, A)$ such that $1_{A}=C l_{\tau}(G)$. For every $a_{x} \widetilde{\in} G$, choose $F_{a_{x}} \in \sigma$ such that $a_{x} \widetilde{\in} F_{a_{x}}$. Then $\left\{F_{a_{x}}: a_{x} \tilde{\in} G\right\}$ is a countable subfamily of $\sigma$. Moreover,

$$
1_{A}=C l_{\tau}(G)=C l_{\tau}\left(\widetilde{\bigcup}\left\{a_{x}: a_{x} \widetilde{\in} G\right\}\right) \widetilde{\subseteq} C l_{\tau}\left(\widetilde{\bigcup}\left\{F_{a_{x}}: a_{x} \widetilde{\in} G\right\}\right) .
$$

and hence $1_{A}=C_{\tau}\left(\widetilde{\cup}\left\{F_{a_{x}}: a_{x} \widetilde{\in} G\right\}\right)$. This shows that $(X, \tau, A)$ is soft weakly Lindelof.

Lemma 8. [6] Let $(X, \tau, A)$ be a STS and $F \in S S(X, A)$. Then for all $a \in A, \overline{F(a)}^{a} \subseteq\left(C l_{\tau}(F)\right)(a)$.

Theorem 42. Let $(X, \tau, A)$ be a STS. If $A$ is countable and $\left(X, \tau_{a}\right)$ is weakly Lindelof for all $a \in A$, then $(X, \tau, A)$ is soft weakly Lindelof.

Proof. Let $\sigma$ be a soft open cover of $(X, \tau, A)$. For each $a \in A,\{F(a): F \in \sigma\}$ is an open cover of $\left(X, \tau_{a}\right)$ and by assumption there is a countable subfamily $\sigma_{a}$ of $\sigma$ such that $\overline{\left.\cup F(a): F \in \sigma_{a}\right\}}{ }^{a}=X$. Let $\gamma=\left\{F: F \in \sigma_{a}, a \in A\right\}$. Then $\sigma_{1}$ is a countable subfamily of $\sigma$. By Lemma 8 , or each $a \in A$,

$$
\begin{aligned}
X & ={\overline{\cup\left\{F(a): F \in \sigma_{a}\right\}}}^{a} \\
& \subseteq \overline{\cup\{F(a): F \in \gamma\}}^{a} \\
& =\overline{(\widetilde{U}\{F: F \in \gamma\})(a)}^{a} \\
& \subseteq\left(C l_{\tau}(\widetilde{\cup}\{F: F \in \gamma\})\right)(a) .
\end{aligned}
$$

It follows that $C l_{\tau}(\widetilde{\cup}\{F: F \in \gamma\})=1_{A}$. Hence $(X, \tau, A)$ is soft weakly Lindelof.

Corollary 17. If $A$ is countable and $\left\{\left(X, \Im_{a}\right): a \in A\right\}$ is a family of weakly Lindelof topological spaces, then $\left(X, \bigoplus_{a \in A} \Im_{a}, A\right)$ is soft weakly Lindelof.

Proof. For each $b \in A$, by Proposition $8,\left(\bigoplus_{a \in A} \Im_{a}\right)_{b}=\Im_{b}$ and so $\left(X,\left(\bigoplus_{a \in A} \Im_{a}\right)_{b}\right)$ is weakly Lindelof. Thus, by Theorem 42 , we have $\left(X, \underset{a \in A}{\bigoplus_{a}} \Im_{a} A\right)$ is soft weakly Lindelof.

The following Example will shows that the converse of Theorem 42 need not to be true in general: 
Example 12. Let $X$ be an uncountable set and let $A$ be a set of parameters contains at least two points. Fix $x \in X$ and $a \in A$. Let $\tau=\left\{0_{A}\right\} \cup\left\{F \in S S(X, A): a_{x} \widetilde{\in} F\right\}$. Then
(a) $(X, \tau, A)$ is a STS.
(b) $(X, \tau, A)$ is soft weakly Lindelof.
(c) $\left(X, \tau_{b}\right)$ is a discrete topological space and hence it is not weakly Lindelof for all $b \neq a$.

Proof. (a) (1) By definition of $\tau, 0_{A} \in \tau$. Also, since obviously $a_{x} \widetilde{\in} 1_{A}$, then $1_{A} \in \tau$.

(2) Let $F, G \in \tau-\left\{0_{A}\right\}$. Then $a_{x} \widetilde{\in} F$ and $a_{x} \widetilde{\in} G$. So $a_{x} \widetilde{\in} F \widetilde{\cap} G$ and hence $F \widetilde{\cap} G \in \tau$.

(3) Let $\left\{G_{\alpha}: \alpha \in \Delta\right\} \subseteq \tau-\left\{0_{A}\right\}$. Then $a_{x} \widetilde{\in} \widetilde{\bigcup}_{\alpha \in \Delta} G_{\alpha}$ and so $\widetilde{\bigcup}_{\alpha \in \Delta} G_{\alpha} \in \tau$.

(b) Let $\sigma$ be a soft open cover of $(X, \tau, A)$. Choose $F \in \sigma-\left\{0_{A}\right\}$ and let $\rho=\{F\}$. Then $\rho$ is a countable subfamily of $\sigma$ with $1_{A}=C l_{\tau}(F)$. It follows that $(X, \tau, A)$ is soft weakly Lindelof.

(c) Straightforward.

Lemma 9. Let $(X, \tau, A)$ be a STS, where $A=\{a\}$. Then $\overline{F(a)}^{a}=\left(C l_{\tau}(F)\right)(a)$.

Proof. Suppose to the contrary that $\overline{F(a)}^{a} \neq\left(C l_{\tau}(F)\right)(a)$. Then by Lemma 8 , there is $x \in$ $\left(C l_{\tau}(F)\right)(a)-\overline{F(a)}^{a}$. So, we have $a_{x} \widetilde{\in} a_{X-\overline{F(a)}} a \in \tau$ and $a_{x} \widetilde{\in} C l_{\tau}(F)$. Thus, $a_{X-\overline{F(a)}} a \widetilde{\cap} F \neq 0_{A}$. Choose $a_{y} \widetilde{\in} a_{X-\overline{F(a)}} a \widetilde{\cap} F$. Then $y \in\left(X-\overline{F(a)}^{a}\right) \cap F(a)$ which is a contradiction.

Theorem 43. Let $(X, \tau, A)$ be a STS, where $A=\{a\}$. Then $(X, \tau, A)$ is soft weakly Lindelof if and only if the topological space $\left(X, \tau_{a}\right)$ is weakly Lindelof.

Proof. Necessity. Suppose that $(X, \tau, A)$ is soft weakly Lindelof. Let $\mathcal{M}$ be an open cover of $\left(X, \tau_{a}\right)$. Then $\left\{a_{M}: M \in \mathcal{M}\right\}$ is a soft open cover of $(X, \tau, A)$ and so there is a countable subfamily $\mathcal{M}_{1} \subseteq \mathcal{M}$ such that $C l_{\tau}\left(\widetilde{\cup}\left\{a_{M}: M \in \mathcal{M}_{1}\right\}\right)=1_{A}$

Sufficiency. Suppose that $\left(X, \tau_{a}\right)$ is weakly Lindelof. Let $\sigma$ be a soft open cover of $(X, \tau, A)$. Then $\{F(a): F \in \sigma\}$ is an open cover of $\left(X, \tau_{a}\right)$ and so there is a countable subfamily $\gamma$ of $\sigma$ such that $\overline{\cup\{F(a): F \in \gamma\}}^{a}=X$. By Lemma 9, we have $\left(C l_{\tau}(\widetilde{\cup}\{F: F \in \gamma\})\right)(a)=\overline{\cup\{F(a): F \in \gamma\}}^{a}=X$. It follows that $C l_{\tau}(\widetilde{\cup}\{F: F \in \gamma\})=1_{A}$. Hence, $(X, \tau, A)$ is soft weakly Lindelof.

Theorem 44. Let $(X, \tau, A)$ be a STS, where $A=\{a\}$. Then $(X, \tau, A)$ is soft separable if and only if the topological space $\left(X, \tau_{a}\right)$ is separable.

Proof. Necessity. Suppose that $(X, \tau, A)$ is soft separable. Then there is $F \in \operatorname{SCSS}(X, A)$ such that $1_{A}=C l_{\tau}(F)$. Since $A=\{a\}$, then there is a countable subset $Y \subseteq X$ such that $F=a_{Y}$. We are going to show that $\bar{Y}^{a}=X$. If $\bar{Y}^{a} \neq X$, then there is $x \in X-\bar{Y}^{a} \in \tau_{a}$. So, there is $G \in \tau$ such that $G(a)=X-\bar{Y}^{a}$ and hence $G=a_{X-\bar{Y}^{a}}$. Since $a_{x} \in G \in \tau$ and $a_{x} \in C l_{\tau}(F)$, then $G \widetilde{\cap} F \neq 0_{A}$. But $G \widetilde{\cap} F=a_{X-\bar{Y}^{a}} \widetilde{\cap} a_{Y}=a_{\left(X-\bar{Y}^{a}\right) \cap Y}=a_{\varnothing}=0_{A}$. It follows that $\bar{Y}^{a}=X$ and hence $\left(X, \tau_{a}\right)$ is separable. Sufficiency. Similar to the necessity part.

The following example shows that the converse of Theorem 40 is not true in general:

Example 13. Let $S$ be the Sorgenfrey line and $(X, \Im)$ be the Cartesian product topological space $S \times S$. It is well known that $(X, \Im)$ is a separable topological space that is not Lindelof. Let $A=\{a\}$ and let $\tau=\left\{a_{U}: U \in \Im\right\}$. Then $(X, \tau, A)$ is a STS with $\tau_{a}=\Im$. Thus, by Theorems 44 and $38(X, \tau, A)$ is soft separable that is not soft Lindelof. Therefore, by Theorem $41(X, \tau, A)$ is soft weakly Lindelof that is not soft Lindelof.

The following example shows that the converse of Theorem 41 is not true in general:

Example 14. Let $X$ be an uncountable set and let $\Im$ be the cocountable topology on X. It is well known that $(X, \Im)$ is a Lindelof topological space that is not separable. Let $A=\{a\}$ and let $\tau=\left\{a_{U}: U \in \Im\right\}$. Then 
$(X, \tau, A)$ is a STS with $\tau_{a}=\Im$. Thus, by Theorems 44 and $38(X, \tau, A)$ is soft Lindelof that is not soft separable. Therefore, by Theorem $40(X, \tau, A)$ is soft weakly Lindelof that is not soft separable.

Lemma 10. Let $(X, \tau, A)$ be a STS and let $\mathcal{B}$ be a soft base of $\tau$. Then $(X, \tau, A)$ is soft weakly Lindelof if and only if every soft open cover $\sigma$ of $(X, \tau, A)$ with $\sigma \subseteq \mathcal{B}$ contains a countable subfamily $\rho$ such that $1_{A}=C l_{\tau}(\widetilde{\cup}\{F: F \in \rho\})$.

Proof. Necessity. It is obvious

Sufficiency. Suppose that every soft open cover $\sigma$ of $(X, \tau, A)$ with $\sigma \subseteq \mathcal{B}$ contains a countable subfamily $\rho$ such that $1_{A}=C l_{\tau}(\widetilde{\cup}\{F: F \in \rho\})$. Let $\sigma$ be a soft open cover of $(X, \tau, A)$ with $\sigma \subseteq \tau-$ $\left\{0_{A}\right\}$. For every $F \in \sigma$, there is $\mathcal{B}_{F} \subseteq \mathcal{B}$ such that $\widetilde{U}\left\{G: G \in \mathcal{B}_{F}\right\}=F$. Let $\mu=\left\{G: G \in \mathcal{B}_{F}, F \in \sigma\right\}$. Then $\widetilde{\cup}\{G: G \in \mu\}=1_{A}$ and by assumption, $\mu$ contains a countable subfamily $\rho$ such that $1_{A}=$ $C l_{\tau}(\widetilde{\cup}\{G: G \in \rho\})$. For each $G \in \rho$, choose $F(G) \in \sigma$ such that $G \widetilde{\subseteq} F(G)$. Then $\{F(G): G \in \rho\}$ is a countable subfamily of $\sigma$. Also,

$$
1_{A}=C l_{\tau}(\widetilde{\bigcup}\{G: G \in \rho\}) \widetilde{\subseteq} C l_{\tau}(\widetilde{\bigcup}\{F(G): G \in \rho\})
$$

which shows that $1_{A}=C l_{\tau}(\widetilde{\bigcup}\{F(G): G \in \rho\})$. It follows that $(X, \tau, A)$ is soft weakly Lindelof.

Theorem 45. Let $(X, \tau, A)$ be a strongly soft anti-locally countable. Then $(X, \tau, A)$ is soft weakly Lindelof if and only if $\left(X, \tau_{s \omega}, A\right)$ is soft weakly Lindelof.

Proof. Necessity. Suppose that $(X, \tau, A)$ is soft weakly Lindelof. By Theorem $18(\mathrm{c}), \tau_{s c}$ is a soft base of $\tau_{s \omega}$, so by Lemma 10 it is sufficient to show that every soft open cover $\sigma$ of $\left(X, \tau_{s \omega}, A\right)$ with $\sigma \subseteq \tau_{s c}$ contains a countable subfamily $\rho$ such that $1_{A}=C_{\tau}(\widetilde{\cup}\{F: F \in \rho\})$. Let $\sigma$ be a soft open cover of $\left(X, \tau_{s \omega}, A\right)$ with $\sigma \subseteq \tau_{s c}$, say $\sigma=\left\{F_{\alpha}-H_{\alpha}: \alpha \in \Delta\right\}$ with $F_{\alpha} \in \tau$ and $H_{\alpha} \in \operatorname{SCSS}(X, \tau)$ for all $\alpha \in \Delta$. Then $\left\{F_{\alpha}: \alpha \in \Delta\right\}$ is a soft open cover of $(X, \tau, A)$. Since $(X, \tau, A)$ is soft weakly Lindelof, then there is a countable subset $\Delta_{1} \subseteq \Delta$ such that $C l_{\tau}\left(\widetilde{\cup}\left\{F_{\alpha}: \alpha \in \Delta_{1}\right\}\right)=1_{A}$ is also a soft open cover of $(X, \tau, A)$. Put $H=\widetilde{\bigcup}\left\{H_{\alpha}: \alpha \in \Delta_{1}\right\}$. Then $H$ is strongly soft countable. For each $a_{x} \widetilde{\in} H$, take $\alpha\left(a_{x}\right) \in \Delta$ such that $a_{x} \widetilde{\in} F_{\alpha\left(a_{x}\right)}-H_{\alpha\left(a_{x}\right)}$. Therefore, $\left\{F_{\alpha}-H_{\alpha}: \alpha \in \Delta_{1}\right\} \cup\left\{F_{\alpha\left(a_{x}\right)}-H_{\alpha\left(a_{x}\right)}: a_{x} \tilde{\in} H\right\}$ is a countable subfamily of $\sigma$ and by Theorem 33,

$$
\begin{aligned}
1_{A} & =C l_{\tau}\left(\widetilde{\bigcup}\left\{F_{\alpha}: \alpha \in \Delta_{1}\right\}\right) \widetilde{\subseteq} C l_{\tau}\left(\left\{F_{\alpha}-H_{\alpha}: \alpha \in \Delta_{1}\right\} \cup\left\{F_{\alpha\left(a_{x}\right)}-H_{\alpha\left(a_{x}\right)}: a_{x} \widetilde{\in} H\right\}\right) \\
& =C l_{\tau_{s \omega}}\left(\left\{F_{\alpha}-H_{\alpha}: \alpha \in \Delta_{1}\right\} \cup\left\{F_{\alpha\left(a_{x}\right)}-H_{\alpha\left(a_{x}\right)}: a_{x} \widetilde{\in} H\right\}\right) .
\end{aligned}
$$

It follows that $\left(X, \tau_{s \omega}, A\right)$ is soft weakly Lindelof.

Sufficiency. It is obvious.

The following example shows in Theorem 45 that the assumption 'strongly soft anti-locally countable' can not be dropped:

Example 15. Let $(X, \tau, A)$ as in Example 12. Then

(a) $\left(X, \tau_{s \omega}, A\right)$ is a discrete STS.

(b) $\left(X, \tau_{s \omega}, A\right)$ is not soft weakly Lindelof.

Proof. (a) We show that $S P(X, A) \subseteq \tau_{s \omega}$. Let $b_{y} \in S P(X, A)$. If $b_{y}=a_{x}$, then $b_{y} \in \tau \subseteq \tau_{s \omega}$. If $b_{y} \neq a_{x}$, then we have $a_{x} \widetilde{\cup} b_{y} \in \tau, a_{x} \in \operatorname{SCSS}(X, A)$ and so $\left(a_{x} \widetilde{\cup} b_{y}\right)-a_{x}=b_{y} \in \tau_{s \omega}$.

(b) By (a), $S P(X, A)$ is a soft open cover of $\left(X, \tau_{s \omega}, A\right)$. If $\rho$ is a countable subfamily of $S P(X, A)$, then by (a) $C l_{\tau_{s \omega}}(\widetilde{\cup}\{F: F \in \rho\})=\widetilde{\cup}\{F: F \in \rho\} \neq 1_{A}$. It follows that $\left(X, \tau_{s \omega}, A\right)$ is not soft weakly Lindelof. 


\section{Conclusions}

In this paper, $\omega$-open sets as a weaker form of open sets in ordinary topological spaces are extended to include soft topological spaces, where soft $\omega$-open sets and strongly soft $\omega$-open sets as two weaker forms of soft open sets are introduced and investigated. The results deal mainly with the relation between the generated soft topology and the given indexed family of topologies, as well as the relation between a given soft topological space and their topological space. Also, as two main results, the soft Lindelofeness and soft weak Lindelofeness are characterized. In future studies, the following topics could be considered: 1 ) To define several types of soft $\omega$-continuity; 2 ) To define separation axioms via soft $\omega$-open sets.

Author Contributions: Formal analysis, investigation, and writing-original draft preparation S.A.G. and W.H. All authors have read and agreed to the published version of the manuscript.

Funding: This research received no external funding.

Conflicts of Interest: The authors declare no conflict of interest.

\section{References}

1. Molodtsov, D. Soft set theory—First results. Global optimization, control, and games. III. Comput. Math. Appl. 1999, 37, 19-31. [CrossRef]

2. Maji, P.K.; Biswas, R.; Roy, A.R. Soft set theory. Comput. Math. Appl. 2003, 45, 555-562. [CrossRef]

3. Kharal, A.; Ahmad, B. Mappings of soft classes. New Math. Nat. Comput. 2011, 7, 471-481. [CrossRef]

4. Molodtsov, D.; Leonov, V.Y.; Kovkov, D.V. Soft sets technique and its application. Nechetkie Sistemy $i$ Myagkie Vychisleniya. 2006, 1, 8-39.

5. Maji, P.K.; Roy, A.R.; Biswas, R. An application of soft sets in a decision making problem. Comput. Math. Appl. 2002, 44, 1077-1083. [CrossRef]

6. Shabir, M.; Naz, M. On soft topological spaces. Comput. Math. Appl. 2011, 61, 1786-1799. [CrossRef]

7. Matejdes, M. Soft topological space and topology on the Cartesian product. Hacet. J. Math. Stat. 2016, 45, 1091-1100.

8. Al-Saadi, H.S.; Min, W.K. On soft generalized closed sets in a soft topological space with a soft weak structure. Int. J. Fuzzy Logic Intell.Syst. 2017, 17, 323-328. [CrossRef]

9. Demir, İ.; Özbakır, O.B.; Yıldız, İ. A contribution to the study of soft proximity spaces. Filomat 2017, 31, 2023-2034. [CrossRef]

10. Hosseinzadeh, H. Fixed point theorems on soft metric spaces. J. Fixed Point Theory Appl. 2017, 19, 1625-1647. [CrossRef]

11. Kandil, A.; Tantawy, O.A.E.; El-Sheikh, S.A.; Hazza, S.A. Some types of pairwise soft sets and the associated soft topologies. J. Intell. Fuzzy Syst. 2017, 32, 1007-1018. [CrossRef]

12. Sayed, O.R.; Hassan, N.; Khalil, A.M. A decomposition of soft continuity in soft topological spaces. Afr. Mat. 2017, 28, 887-898. [CrossRef]

13. Zakari, A.H.; Ghareeb, A.; Omran, S. On soft weak structures. Soft Comput. 2017, 21, 2553-2559. [CrossRef]

14. Thakur, S.S.; Rajput, A.S. Connectedness between Soft Sets. New Math. Natural Comp. 2018, 14, 53-71. [CrossRef]

15. Tahat, M.K.; Sidky, F.; Abo-Elhamayel, M. Soft topological soft groups and soft rings. Soft Comput. 2018, 22, 7143-7156. [CrossRef]

16. Kandemir, M.B. The concept of $\sigma$-algebraic soft set. Soft Comput. 2018, 22, 4353-4360. [CrossRef]

17. Ozturk, T.Y.; Gunduz Aras, C.; Yolcu, A. Soft bigeneralized topological spaces. Filomat 2018, 32, 5679-5690. [CrossRef]

18. El-Shafei, M.E.; Abo-Elhamayel, M.; Al-shami, T.M. Partial soft separation axioms and soft compact spaces. Filomat 2018, 32, 4755-4771. [CrossRef]

19. Polat, N.Ç.; Yaylalı, G.; Tanay, B. A new approach for soft semi-topological groups based on soft element. Filomat 2018, 32, 5743-5751. [CrossRef]

20. Abbas, M.; Murtaza, G.; Romaguera, S. Remarks on fixed point theory in soft metric type spaces. Filomat 2019, 33, 5531-5541. [CrossRef] 
21. Al Ghour, S.; Bin-Saadon, A. On some generated soft topological spaces and soft homogeneity. Heliyon 2019, 5, e02061. [CrossRef] [PubMed]

22. Polat, N.Ç.; Yaylalı, G.; Tanay, B. Some results on soft element and soft topological space. Math. Methods Appl. Sci. 2019, 42, 5607-5614. [CrossRef]

23. Al-shami, T.M.; El-Shafei, M.E.; Abo-Elhamayel, M. On soft topological ordered spaces. J. King Saud Univ.-Sci. 2019, 31, 556-566. [CrossRef]

24. Al-Omari, A. Soft topology in ideal topological spaces. Hacet. J. Math. Stat. 2019, 8, 1277-1285. [CrossRef]

25. Kiruthika, M.; Thangavelu, P. A link between topology and soft topology. Hacet. J. Math. Stat. 2019, 48, 800-804. [CrossRef]

26. Gunduz Aras, C.; Bayramov, S.; Yazar, M.I. Soft $d$-metric spaces. Bol. Soc. Parana. Mat. 2020, 38, $137-147$. [CrossRef]

27. Hdeib, H.Z. $\omega$-closed mappings. Rev. Colombiana Mat. 1982, 16, 65-78.

28. Hdeib, H.Z. $\omega$-continuous functions. Dirasat J. 1989, 16, 136-142.

29. Al-Omari, A.; Noorani, M.S.M. Contra- $\omega$-continuous and almost Contra- $\omega$-continuous. Int. J. Math. Math. Sci. 2007, 40469. [CrossRef]

30. Al-Omari, A.; Noiri, T.; Noorani, M.S.M. Weak and strong forms of $\omega$-continuous functions. Int. J. Math. Math. Sci. 2009, 174042. [CrossRef]

31. Al Ghour, S. Some generalizations of paracompactness. Missouri J. Math. Sci. 2006, 18, 64-77. [CrossRef]

32. Al-Zoubi, K.Y. On generalized $\omega$-closed sets. Int. J. Math. Math. Sci. 2005, 13, 2011-2021. [CrossRef]

33. Al-Hawary, T.; Al-Omari, A. Between open and $\omega$-open sets. Questions Answers Gen. Topol. 2006, $24,67-78$.

34. Al-Hawary, T.; Al-Omari, A. Decompositions of continuity. Turkish J. Math. 2006, 30, 187-195.

35. Al-Omari, A.; Noorani, M.S.M. Regular generalized $\omega$-closed sets. Int. J. Math. Math. Sci. 2007, 16292.

36. Sarsak, M. A decomposition of $\omega$-almost Lindelöf spaces. Questions Answers Gen. Topol. 2009, 27, $137-139$.

37. Al Ghour, S.; Irshedat, B. The topology of $\theta_{\omega}$-open sets. Filomat 2017, 31, 5369-5377.

38. Al Ghour, S. Theorems on strong paracompactness of product spaces. Math. Notes 2018, 103, 54-58. [CrossRef]

39. Al-Omari, A.; Al-Saadi, H. A topology via $\omega$-local functions in ideal spaces. Mathematica 2018, 60, $103-110$. [CrossRef]

40. Carpintero, C.; Rajesh, N.; Rosas, E. On real valued $\omega$-continuous functions. Acta Univ. Sapientiae Math. 2018, 10, 242-248. [CrossRef]

41. Noble, N. Some thoughts on countable Lindelöf products. Topol. Appl. 2019, 259, 287-310. [CrossRef]

42. Al Ghour, S.; El-Issa, S. $\theta_{\omega}$-Connectedness and $\omega$ - $R_{1}$ properties. Proyecciones 2019, 38, 953-974. [CrossRef]

43. Rosas, E.; Carpintero, C.; Rajesh, N.; Shanti, S. Near $\omega$-continuous multifunctions on bitopological spaces. Proyecciones 2019, 38, 691-698. [CrossRef]

44. Mahanta, J.; Das, P.K. On soft topological space via semiopen and semiclosed soft sets. Kyungpook Math. J. 2014, 54, 221-236. [CrossRef]

45. Akdag, M.; Ozkan, A. Soft b-open sets and soft b-continuous functions. Math. Sci. (Springer) 2014, 8, 124. [CrossRef]

46. Akdag, M.; Ozkan, A. Soft $\alpha$-open sets and soft $\alpha$-continuous functions. Abstr. Appl. Anal. 2014, 891341.

47. Kandil, A.; Tantawy, O.A.E.; El-Sheikh, S.A.; Abd El-latif, A.M. Soft semi compactness via soft ideals. Appl. Math. Inf. Sci. 2014, 8, 2297-2306. [CrossRef]

48. Kandil, A.; Tantawy, O.A.E.; El-Sheikh, S.A.; Abd El-latif, A.M. Soft semi separation axioms and some types of soft functions. Ann. Fuzzy Math. Inform. 2014, 8, 305-318.

49. Al-shami, T.M.; El-Shafei, M.E.; Abo-Elhamayel, M. Almost soft compact and approximately soft Lindelöf spaces. J. Taibah Univ. Sci. 2018, 12, 620-630. [CrossRef]

50. Al-shami, T.M.; El-Shafei, M.E. Two types of separation axioms on supra soft topological spaces. Demonstr. Math. 2019, 52, 147-165. [CrossRef]

51. Zorlutuna, I.; Akdag, M.; Min, W.K.; Atmaca, S. Remarks on soft topological spaces. Ann. Fuzzy Math. Inform. 2012, 3, 171-185.

52. Das, S.; Samanta, S.K. Soft metric. Ann. Fuzzy Math. Inform. 2013, 6, 77-94.

53. Çağman, N.; Karataş, S.; Enginoglu, S. Soft topology. Comput. Math. Appl. 2011, 62, 351-358. [CrossRef]

54. Nazmul, S.; Samanta, S.K. Neighbourhood properties of soft topological spaces. Ann. Fuzzy Math. Inform. 2012, 6, 1-15. 
55. Terepeta, M. On separating axioms and similarity of soft topological spaces. Soft Comput. 2019, 23, $1049-1057$. [CrossRef]

56. Hussain, S.; Ahmad, B. Soft separation axioms in soft topological spaces. Hacet. J. Math. Stat. 2015, 44, 559-568. [CrossRef]

57. Hussain, S.; Ahmad, B. Some properties of soft topological spaces. Comput. Math. Appl. 2011, 62, 4058-4067. [CrossRef]

58. Aygünoglu, A.; Aygün, H. Some notes on soft topological spaces. Neural Comput. Appl. 2012, 21, 113-119. [CrossRef]

59. Rong W. The countabilities of soft topological spaces, World Accedmy Sci. Eng. Technol. 2012, 6, 784-787.

60. Hart, K.P.; Nagata, J.-I.; Vaughan, J.E. (Eds.) Encyclopedia of General Topology; Elsevier Science Publishers: Amsterdam, The Netherland, 2004; $x+526 p$.

(C) 2020 by the authors. Licensee MDPI, Basel, Switzerland. This article is an open access article distributed under the terms and conditions of the Creative Commons Attribution (CC BY) license (http://creativecommons.org/licenses/by/4.0/). 